\title{
A Nonlinear Model of the Business Cycle*
}

\author{
Edward E. Leamer and Simon M. Potter
}

\author{
Preliminary and Incomplete \\ Please Do Not Quote Without Permission. \\ September 2002
}

\begin{abstract}
A bstract
The usual index of leading indicators has constant weights on its components and is therefore implicitly premised on the assumption that the dynamical properties of the economy remain the same over time and across phases of the business cycle. We explore the possibility that the business cycle has phases, for example, recessions, recoveries and normal growth, each with its unique dynamics. Based on this possibility we develop a nonlinear model of the business cycle that combines a number of previous approaches. We model the state of the economy as a latent variable with a threshold autoregression structure. In addition to dependence on its own lags the latent variable is also determined by observed economic and financial variables. In turn these variables are modeled as following a nonlinear vector autoregression with regimes defined by the latent business cycle variable. A Markov Chain Monte Carlo algorithm is developed to estimate the model. Special attention is paid to specification of prior distributions given the large dimension of the model. We also investigate using the business cycle chronology of the NBER to aid in the classification of the latent variable. The two main empirical objectives of the model
\end{abstract}

*Leamer: Anderson Graduate School of Management, UCLA. Potter: Federal Reserve Bank of New York.The views expressed in this paper are those of the authors and do not necessarily reflect the views of the Federal Reserve Bank of New York or the Federal Reserve System. 
are to provide more accurate predictions of economic variables particularly at turning points and to describe how the dynamics differ across business cycle phases.

\section{Introduction}

We explore the possibility that the business cycle has phases, for example, recessions, recoveries, productivity surges, fragile growth and normal growth, each with its unique dynamics. To aid us with the exploration we develop a nonlinear model of the business cycle that combines and extends a number of previous approaches. We have two objectives: first to provide more accurate predictions of business cycle turning points and thus economic variables in general; second to describe how the dynamics of economic variables change over the phases of the business cycle and over time.

Why would such an exploration be interesting. From the dominant linear perspective such an exploration is distinctly uninteresting. From this perspective, well captured by the use of linear VARs or linear common factor models, knowledge of the current business cycle phase gives no additional information for predicting the future. Of course if one takes such models and enters business cycle expansions and recessions as dummy variables one usually finds significant effects. The explanation for this result from the linear perspective is the perfectly reasonable one that recessions reflect the presence of large negative shocks and expansions the absence of large negative shocks. The NBER dates are produced ex-post and contain information on these large negative shocks. That is, it is not surprising that recessions have lower rates of economic activity than expansions. However, these large negative shocks are not predictable ex-ante.

Another (mildly nonlinear) perpective accepts that dynamics differ between recession and expansions and possibly also recovery periods. Indeed nearly all of the work on nonlinear time series has focused on the difference between recession and expansions in the post-World War II time period. There are three main findings: the move from expansion to recession is abrupt (Hamilton ,1989); recessions have larger shocks than expansions (Koop and Potter 2001); there is some bounce-back in terms of higher growth in the recovery period after a recession (Beaudry and Koop, 1993, Potter, 1995). The first of these results essentially agrees with the linear perspective; the 
second states there is higher order dependence in the shocks hitting the economy that will affect forecast distributions but not point forecasts. Only the bounce-back result (see also Sichel 1994), also known as plucking theory of business cycles, is in direct contradiction to the linear perspective.

Recession and recovery periods make up less than one third of the time periods since 1945 and since 1982 only about one fifth of the periods. Consider also that the average length of expansions is 57 months but the range is from 12 months to 120 months with only the late 1970s expansion being close to the average. In contrast recessions are much more homogeneous in their lenght ranging from 6 to 16 months. What accounts for the differing lengths of expansions?

Leamer (2001) investigates whether there is a life-cycle to an expansion. Unlike the previous research on duration dependence in recessions and expansions, he focuses on the three long expansions of the 1960s, 1980s and 1990s and attempts to analyze the sources of the "spurt" in growth that occured as these expansions continued past 4 years. Further, unlike previous work on forecasting recessions, Leamer focuses on the period immediately before business cycle peaks to see if this might be modeled as a different type of phase (we will call it the fragile one) and transitions to it predicted by a specific set of indicators. He finds that hours, unemployment and profits are good predictors of transitions into this fragile phase.

Leamer's exploration was mainly guided by the use of "spider" charts that show the path of the same variable across different business cycle phases, with their levels normalized to be equal at the start of the phase. Such charts are frequently used to informally predict the behavior of variables over a particular phase such as a recession or to attempt to classify where a particular phase is presently occuring. Such an approach is solidly in the NBER tradition of Burns and Mitchell's and makes little sense from the dominant linear perspective. Thus, it is impossible to formalize such an approach using linear methods. Further, the inference from spider charts relies heavily on the judgement of the investigator and the choice of business cycle phase start dates.

Consider the "stylized fact" that the stock market has predicted 9 of the last 5 recessions. A spider chart for the behavior of stock market indices around business cycle peaks shows a pattern of a decline before the peak and then a turn-around before the trough. However, the stock market also has large declines without being followed by a business cycle peak and the behavior around actual peaks is very varied. In contrast, consider the unem- 
ployment rate. It tends to increase mildly just before a business cycle peak but then increase very strongly after the peak. The behavior is much more uniform than the stock market around peaks and crucially the unemployment rate has no increases of more than 0.4 percentage points without a recession occurring. Of course once a large jump in the unemployment rate occurs the recession has probably already started. Further, the smallest increase in unemployment during a period including a recession is 2 percentage points, thus it is possible that a smaller increase, for example 1 percentage point might not be associated with a recession.

The model we develop provides a means of formalizing the information contained in spider type charts for a large number of variables and at the same time provides a statistical method for dating business cycle phases. Returning to the ambiguity of the stock market as a leading indicator of recessions and the precision of increases in the unemployment rate as an indicator of recessions. In the nonlinear model we construct the stock market would indicate the possible move into a fragile phase of the business cycle. This transition would be confirmed if the unemployment rate remained constant or increased slightly but would be downweighted if the unemployment rate declined. Forecasts of economic variables following the initial decline in the stock market would place an increased weight on the behavior of the variables in fragile phases and on the downturn phase that would be more likely to occur after the fragile phase. Even if the only difference in behavior across phases was differing levels of uncertainty this would affect the forecast distribution. The weight on the behavior of the fragile phase would change as new data on the unemployment rate and stock market arrived. For example, suppose the stock market decline is quickly reversed (as in the Fall of 1998) and the unemployment rate continues to decline then this would make the fragile followed by recession phase less likely than the initial assessment following the stock market fall.

Perhaps the most outstanding feature of the US economy in the last 20 years is its increased stability. From the perspective of the model we develop we are able to give a different interpretation than the standard linear ones of smaller shocks or better inventory management. Since the volatility of economic variables is different across business cycle phases, longer durations in less volatile phases would produce an overall decrease in volatility. 


\subsection{Literature R eview}

The empirical analysis of business cycles has two distinct strands (see the review article by Diebold and Rudebusch 1996): the classification approach of the NBER originating with Burns and Mitchell and the econometric modeling approach originating with the Cowles Commission. One interpretation of the model developed in this paper is that it allows insights from the classification approach to assist in the construction of an econometric model. We are not the first to try to combine the classification and econometric approach (see Diebold and Rudebusch 1996 and 2001 for comprehensive literature reviews).

The closest in spirit to our approach is the research prompted by Hamilton's 1989 Markov Switching model of US Output, particularly the papers of Diebold, Lee and Weinbach (1996) and Filardo (1994), (DLW\&F hereafter). In the Markov switching approach there are usually 2 or 3 business cycle phases that are modeled as the states of an unobserved Markov chain. In Hamilton's original paper the transitions between the states were exogenous and had a constant hazard. In the extensions by DLW\&F the probability of transition was allowed to depend on various leading indicators but the evolution of these indicators over the cycle is not modeled, unlike the approach we pursue.

Another branch of the literature are dynamic factor models, the best known example is Stock and Watson's (1989, 1991 and 1993) (SW hereafter) dynamic factor approach. In their early work SW used statistical methods to extract a dynamic factor from the Commerce Department's list of 4 coincident indicators and used various leading indicators to predict future values of the dynamic factor. In particular they developed a sophisticated recession probability index based on the forecast distribution of the dynamic factor. Chauvet (1998) combines the approach of Stock and Watson with that of Hamilton, following suggestions by Diebold and Rudebusch (1996) that such a model would integrate the classification and econometric approach. This factor model with Markov switching does a good job of tracking the recession starting in March 2001 (see Chauvet 2002). In more recent work, Stock and Watson (2002) and Knox, Stock and Watson (2000) have developed methods that allow many more variables to be used to produce the dynamics factors. There is some indication (Watson 2002) that such a "large data" approach could pay dividends in forecasting performance. In the construction of our model we will also exploit common factors restrictions with the flavor of leading and coincident indicators but similar to Chauvet these common factors 
will be constructed for individual phases of the business cycle.

A somewhat separate literature focuses on probit modeling for recession prediction. Perhaps the best known paper is Estrella and Mishkin (1998) where they find that although a range of leading indicators are statistically significant in sample, only the term spread is useful in genuine forecasting exercises. Our model for the business cycle latent variable is very similar to that proposed by Chauvet and Potter (2002). In a similar manner Dueker (2001) has extended the probit approach by allowing for an unobserved business cycle variable tracking the NBER dates to be used in a standard VAR. The main innovation in Dueker as in our work is that the variables determining the business cycle phase are endogenously determined and thus their behavior can be forecast out of the sample period.

The models described above required a number of advances in econometric technique and computational speed. Nelson and Kim 's 1999 monograph provides an excellent introduction to all of these models and a description of best practice classical and Bayesian techniques for their estimation.

\subsection{Outline of Paper}

The model we develop is very complex in terms of the number of latent variables involved and and large number of unknown parameters. Each part of the model is relatively simple which allows us to construct a straightforward Gibbs sampling alogorithm for its estimation. The standard parts of the estimation are described in the appendix. Some of the non-standard aspects are described in detail in the main text.

We start in Section 2.1 by describing a threshold autoregressive model for

the latent variable determining business cycle phases. The exact definition of the business cycle phases are left to Section 3 . The threshold autoregressive model uses information from variables modeled by a VAR described in the rest of this section in a phase dependent way. In Section 2.2 we discuss how the variables helping determine the phase loosely fit into two categories: leading or coincident indicators. The usefulness of a particular indicator can vary across phases. Since we want to allow for a large number of time series to predict the business cycle latent variable we introduce a common factor structure into a linear VAR in Section 2.3. This requires the estimation of a further set of latent variables in the Gibbs sampler. Section 2.4 generalizes the VAR developed in Sections 2.2 and 2.3 to one with a threshold structure with regimes defined by business cycle phases. 
In section 2.4.1 we detail one of the main advantages of our model over previous one. We show how conditioning on information about business cycle phases, we can use the differing relationship of leading and coincident indicators in and across business cycle phases to improve forecasts of future values.

In Section 3 we turn to the definition and estimation of business cycle phases. Section 3.1 provides 2 definitions of business cycles. The definitions differ on how recovery phases are treated. The subsection also discusses possible sources of increased stability in the business cycle. Subsection 3.1.1 discusses some of the similarities with Markov switching models and highlights some of the differences. Section 3.2 discusses how the model itself can be directly used to identify business cycle phases. It provides a detailed description of the block of the Gibbs sampler that generates the business cycle phases. Section 3.3 describes ways of directly introducing the NBER dates into the estimation of business cycle phases.

Since the model is so complex it is esssential to evaluate the model with its predictions for new data. Of course one advantage is that the model is designed to produce lots of predictions in real time. Section 4.1 provides a description of forecasting and dynamic simulation of the model. Section 4.2 considers difficulties associated with constructing forecast of recoveries and trough dates. Section 4.3 contrasts our approach to forecasting recession phases with that of Estrella and Mishkin (1998).

Section 5.1 gives an overview of the Gibbs sampler. The detailed information on the sampler is contained in the appendix. Section 5.2 contains information on the prior distributions used for the business cycle latent variable model. Section 5.3 discusses various approaches to constructing prior distributions for the nonlinear VAR and the common factor structure contained within it.

Section 6 contains a small scale application to the unemployment rate and term spread. There are some initial results for this application available. Section 7 will contain a large scale applications to the leading and coincident indicators of the Conference Board. Section 8 concludes.

\section{Statistical Model}

Our statistical model has two components: a latent variable model for determining business cycle phases and a vector autoregressive model for observ- 
able variables. Both models have a threshold autoregressive structure with regimes defined by phases of the business cycle. We leave the determination of these phases/regimes to the next section.

Time is indexed by the integer $t \in \mathcal{T}$. The set $\mathcal{T}$ can be decomposed into 5 disjoint subsets $\mathcal{T}=R \cup N \cup S \cup F \cup D$, where $D$ represents periods of downturns, $R$ represents periods of recovery from downturns, $N$ represents periods of normal growth, $S$ represents periods when growth spurts and $F$ represents periods of fragile growth. $\mathcal{T}$ can also be into split into subsets representing particular "business cycles" although as we discuss below such a decomposition might have a union smaller than $\mathcal{T}$. We denote the current business cycle or most recent business cycle by J.(Burns and Mitchell had 9 phases we have 5).

\subsection{Model for Latent Variable}

We define a latent stochastic process $Z_{t}$. This latent variable will be used to classify the phase of the business cycle according to a set of rules defined in Section 3. For the moment, we ignore the exact description of these rules. $Z_{t}$ follows a threshold autoregressive structure where the conditional mean is determined by the business cycle phase in the the last period along with a phase specific set of observable variables and the conditional variance is time dependent.

$$
Z_{t}=\left\{\begin{array}{c}
X_{N t}+\theta_{N} Z_{t-1}+\sigma(t) \epsilon_{t}, \text { if } t-1 \in N \\
X_{R t}+\theta_{R} Z_{t-1}+\sigma(t) \epsilon_{t}, \text { if } t-1 \in R \\
X_{S t}+\theta_{S} Z_{t-1}+\sigma(t) \epsilon_{t}, \text { if } t-1 \in S \\
X_{F t}+\theta_{F} Z_{t-1}+\sigma(t) \epsilon_{t}, \text { if } t-1 \in F \\
X_{D t}+\theta_{D} Z_{t-1}+\sigma(t) \epsilon_{t}, \text { if } t-1 \in D
\end{array}\right.
$$

where $\epsilon_{t} \sim \operatorname{IIDN}(0,1), X_{p t}$ are random variables defined by $\left\{\alpha_{P}+\boldsymbol{\beta}_{P}^{\prime} \mathbf{Y}_{t}, P=\right.$ $1, \ldots 5\}$, with $\boldsymbol{\beta}_{P}$ a $M \times 1$ vector of parameters and $\mathrm{Y}_{t}$ is $M \times 1$ vector of economic variables realized at time $t,\{\sigma(t)\}$ is a time varying innovation variance. Below we will parameterize time variation as relating to individual business cycles.

In describing the MCMC algorithm below we will denote the complete set of parameters for the latent variables by $\Psi$. We decompose $\Psi$ into four 
sets $\left\{\Psi_{i}: i=1, \ldots, 4\right\}$ with elements

$$
\begin{aligned}
& \boldsymbol{\psi}_{1}=\left[\alpha_{P}, \boldsymbol{\beta}_{P}^{\prime}\right]^{\prime}, P=1, \ldots, 5 \\
& \boldsymbol{\psi}_{2}=\left[\theta_{1}, \ldots, \theta_{5}\right]^{\prime}, \\
& \boldsymbol{\psi}_{3}=[\sigma(1), \ldots, \sigma(J), \sigma(J+1)]^{\prime}, \\
& \boldsymbol{\psi}_{4}=\left[\underline{v}, \underline{v s^{2}}\right]^{\prime}
\end{aligned}
$$

where we include $\sigma(J+1)$ to allow for fact that a new business cycle might start during the estimation period as described below. The fourth subset $\Psi_{4}$ contains the parameters of the model for determining the prior distributions on the elements of $\Psi_{3}$ which potentially we might also update on in a standard heiriachical manner.

\subsection{M odel for Observable Economic Variables}

The model for business cycle classification contains information from the random vector $\mathbf{Y}_{t}$. We think of the vector as containing information from two distinct type of variables: business cycle leading indicators, mainly financial variables, and coincident macroeconomic aggregates such as GDP, consumption, employment, hours and industrial production. Further, some of these coincident variables will serve the role of phase classifiers and some will serve the roll of indicating transitions between phases in the near future.

We denote the total number of distinct variables in $\mathrm{Y}_{t}$ by $K=K_{L}+K_{C}$ where $K_{L}$ is the distinct number of leading indicators and $K_{C}$ is the number

of distinct coincident indicators. In general the length of the vector $Y_{t}$ will be substantially greater than $K$ since we will require it to equal to the maximum of

- The minimum state vector for describing the dynamics of $K$ unique variables in $Y_{t}$ across the business cycle phases.

- The maximum lead length amongst the $K_{L}$ leading indicators plus 1.

Consider limiting ourselves to one leading indicator $L_{t}$, one coincident aggregate $C_{t}$. Assume the leading indicator leads by one period and that the minimum state vector is $\left[C_{t}, L_{t}, C_{t-1}, L_{t-1}\right]$, i.e., a second order Markov 
assumption. Then consistent with the concept of leading and coincident indicators we could have:

$$
\mathbf{Y}_{t}=\left[\begin{array}{c}
C_{t} \\
L_{t} \\
C_{t-1} \\
L_{t-1}
\end{array}\right], \beta_{P}=\left[\begin{array}{c}
\beta_{P C} \\
0 \\
0 \\
\beta_{P L}
\end{array}\right], P=1, \ldots 5 .
$$

The idea is to include not only leading indicators but also coincident indicators to help in the classification of business cycle phases and the transitions among them. Consider the stylized fact that the stock market has predicted 9 of the last 5 recessions. In our set up the leading indicator might be suggesting a phase transition but unless confirmed by the coincident variable's behavior, the prediction would at best tentative.

We now turn to a model for the evolution of $\mathrm{Y}_{t}$ over time. Although we are mainly interested in a nonlinear model for this evolution we start by discussing a standard linear model.

\subsubsection{Linear Model}

Continuing with the simple example of one leading indicator and one coincident indicator, assume that the model for this bivariate system is constant across business cycle phases and is given by the companion form of a $\operatorname{VAR}(2)$ :

$$
\mathrm{Y}_{t}=\mathrm{a}+\mathrm{A} \mathrm{Y}_{t-1}+\mathrm{HV}_{t}
$$

where the $4 \times 4$ companion matrix $A$ is given by

$$
\left[\begin{array}{ll}
\Phi_{1} & \Phi_{2} \\
l_{2} & 0_{2}
\end{array}\right],
$$

$\Phi_{1}, \Phi_{2}$ are $2 \times 2$ matrices, a is a $4 \times 1$ vector of the form $\left[a_{C}, a_{L}, 0,0\right]^{\prime}$, the $4 \times 2$ matrix $\mathrm{H}$ is given by

$$
\left[\begin{array}{l}
\mathrm{I}_{2} \\
\mathrm{O}_{2}
\end{array}\right]
$$

$\mathrm{V}_{t}$ is a $2 \times 1$ Gaussian random vector with mean zero and variance $\Sigma$.

A strict interpretation of the leading indicator versus coincident indicator would be that $\Phi_{1}[2,1]=\Phi_{2}[2,1]=0$ (the coincident indicator does not help 
predict the leading indicator). Further, one might expect that $\Phi_{1}[1,2], \Phi_{2}[1,2]$ were non-zero (the leading indicator helps predict the coincident indicator) and that if the leading indicator was a financial variable that $\Phi_{1}[2,2]=1$ or 0 (efficient markets type assumption), and $\Phi_{2}[2,2]=0$.

The forecast distributions of this linear state space model are multivariate Gaussian with mean:

$$
\begin{array}{r}
E_{T}\left[\mathbf{Y}_{T+h}\right]=\left(\mathbf{I}+\mathbf{A}+\cdots+\mathbf{A}^{h-1}\right) \mathbf{a}+\mathbf{A}^{h} \mathbf{Y}_{T} \text { and variance: } \\
\operatorname{Var}_{T}\left[\mathbf{Y}_{T+h}\right]=\left(\Sigma+\mathbf{A} \Sigma \mathbf{A}^{\prime}+\cdots+\mathbf{A}^{h-1} \Sigma \mathbf{A}^{h-1 \prime}\right) .
\end{array}
$$

These forecast distributions do not depend on the phase of the business cycle but can be used directly to construct forecast distributions for $Z_{T}$ conditional on a particular phase (or sequence of phases).

$$
\begin{aligned}
& E\left[Z_{T+l} \mid T+\ell \in P, \ell=0, \ldots L-1\right] \\
= & \sum_{\ell=0}^{L} \theta_{P}^{L-\ell}\left(\alpha_{P}+\boldsymbol{\beta}_{P}^{\prime} E_{T}\left[\mathbf{Y}_{T+l}\right]\right)+\theta_{P}^{L} Z_{T}, \\
& \operatorname{Var}_{T}\left[Z_{T+L}\right] \\
= & \sum_{\ell=0}^{L} \theta_{P}^{2(L-\ell)}\left(\sigma^{2}(T+L-\ell)+\boldsymbol{\beta}_{P}^{\prime} \operatorname{Var}_{T}\left[\mathbf{Y}_{T+\ell}\right] \boldsymbol{\beta}_{P}\right) .
\end{aligned}
$$

\subsection{Common Factor R estrictions}

In practice there are literally hundreds of variables which one could use as leading or coincident indicators. Even without allowing the dynamics of the VAR to change over the phases of the cycle, the number of parameters to be estimated would be many times larger than the sample size. The main solution to this dimensionality problem is to assume that the dynamics are well described by a few common factors. In other words, the business cycle is driven by just a few shocks rather than the hundreds of shocks defined by an unrestricted vector autoregression.

In our case we decompose the vector $\mathrm{Y}_{t}$ into $k$ observed economic variables $\mathrm{y}_{t}$ and $s$ common factors, $\mathrm{cf}_{t}$ with the following specification:

$$
\begin{aligned}
\mathrm{y}_{t} & =\partial+\Pi(B) \mathrm{y}_{t-1}+\Lambda(B) \mathbf{c f}_{t}+\mathrm{u}_{t}, \\
\mathbf{c f}_{t} & =\mathrm{b}+\Theta(B) \mathbf{c f}_{t-1}+\mathbf{w}_{t}
\end{aligned}
$$


where $\supset$ is a $k \times 1$ vector, $\Pi(B)$ is a diagonal back operator polynomial matrix of order $q, \Lambda(B)$ is a $k \times s$ matrix in the back operator polynomial also of order $q, \mathrm{u}_{t}$ is a $k \times 1$ Gaussian random vector with diagonal variance matrix $\Sigma^{\mathrm{u}}, \mathrm{b}$ is a $s \times 1$ vector, $\Theta(B)$ is a $s \times s$ matrix polynomial of order $q$ in the back operator and $\mathbf{w}_{t}$ is a $s \times 1$ Gaussian random vector with variance matrix $\Sigma^{\mathrm{w}}$. Although the order of the back operator polynomials is assumed to be $q$, some of the matrices might be null at higer orders. Below we discuss methods to estimate the common factors from the observables but one could also include in the list of common factors important observables such as an overall measure of activity or the term spread (Bernanke, Boivin and Elotz (2002) call this the Factor Augmented Vector Autogression). In an empirical example in Section 7 we use the published leading and coincident indices.

Then stacking the observables and common factors in a state space form as above we have

$$
\begin{aligned}
\mathbf{Y}_{t}= & {\left[\begin{array}{c}
\mathrm{y}_{t} \\
\mathrm{cf}_{t} \\
\vdots \\
\mathrm{y}_{t-q+1} \\
\mathrm{cf}_{t-q+1}
\end{array}\right]=\left[\begin{array}{c}
\partial+\Lambda_{1} \mathrm{~b} \\
\mathrm{~b} \\
0_{(q-1)(s+k)}
\end{array}\right]+} \\
& {\left[\begin{array}{cc}
\Pi & \Lambda \Theta \\
0_{s} & \Theta \\
\mathrm{A}_{31} & 0 \\
0 & \mathrm{~A}_{42}
\end{array}\right] \mathrm{Y}_{t-1}+\left[\begin{array}{cc}
\mathrm{I}_{k} & \Lambda_{1} \\
0_{s} & \mathrm{I}_{s} \\
0_{(q-1)(s+k) \times k} & 0_{(q-1)(s+k) \times s}
\end{array}\right]\left[\begin{array}{c}
\mathrm{U}_{t} \\
\mathbf{W}_{t}
\end{array}\right], }
\end{aligned}
$$

where

$$
\Lambda \Theta=\left[\begin{array}{llll}
\Lambda_{1} \Theta_{1} & \Lambda_{1} \Theta_{2}+\Lambda_{2} & \cdots & \Lambda_{1} \Theta_{q}+\Lambda_{q}
\end{array}\right]
$$

and $\Pi=\left[\begin{array}{lll}\Pi_{1} & \cdots & \Pi_{q}\end{array}\right]$.

\subsection{General Nonlinear Model}

The linear model described above embodies the restriction that dynamics are invariant across the business cycle. This restriction certainly makes prediction of business cycles computationally easier but it is a strong restriction. We relax it by assuming that the evolution of the random vector $Y_{t}$ is governed by a nonlinear vector autoregression. The nonlinearity is of a regime 
switching type with the same regime classification as for the latent variable $Z_{t}$.

The description of the general nonlinear model in state space form is:

$$
\mathbf{Y}_{t}=\left\{\begin{array}{c}
\mathrm{a}_{N}+\mathrm{A}_{N} \mathrm{Y}_{t-1}+\mathrm{HV}_{N t} \text { if } t-1 \in N \\
\mathrm{a}_{R}+\mathrm{A}_{R} \mathrm{Y}_{t-1}+\mathrm{HV}_{R t} \text { if } t-1 \in R \\
\mathrm{a}_{S}+\mathrm{A}_{S} \mathrm{Y}_{t-1}+\mathrm{HV}_{S t} \text { if } t-1 \in S \\
\mathrm{a}_{F}+\mathrm{A}_{F} \mathrm{Y}_{t-1}+\mathrm{HV}_{F t} \text { if } t-1 \in F \\
\mathrm{a}_{D}+\mathrm{A}_{D} \mathrm{Y}_{t-1}+\mathrm{HV}_{D t} \text { if } t-1 \in D
\end{array},\right.
$$

where, $\left\{\mathrm{a}_{P}, P=1, \ldots 5\right\}$ are $M \times 1$ vectors, $\left\{\mathrm{A}_{P}, P=1, \ldots 5\right\}$ are $M \times M$ matrices, $\mathrm{H}$ is an $M \times K$ matrix and $\left\{\mathrm{V}_{P t}, P=1, \ldots 5\right\}$ is a $K \times 1$ random vectors distributed independent, multivariate normal distribution with mean vector zero, covariance matrix $\left\{\Sigma_{P}, P=1, \ldots 5\right\}$ and are independent of $\epsilon_{t}$.

The forecast distributions generated by this nonlinear model are also conditionally Gaussian given a future path of the business cycle phases.

To reduce the dimensionality of the parameter space further we only allow certain of the sub-vectors of $\mathrm{a}_{P}$ and submatrices of $\mathrm{A}_{P}$ and $\Sigma_{P}$ to vary with the phase. The most parsimonious version would have all the changes across the phases determined by the common factor model $\left(\mathrm{b}_{P}, \Theta_{P}, \Sigma_{P}^{\mathrm{w}}\right)$. In addition one could limit the changes in non-recovery expansion periods to the intercepts and variance matrix of the innovations of the common factor model.

\subsubsection{Updating Information on Unknown Variables in the VAR}

Suppose that time $T-1$ is known to be a normal expansion phase but, in contrast to the known value of the leading indicator at time $T$, the coincident variable is only known with a lag. Then, just using the information from the current value of the leading indicator, the conditional distribution for $C_{T}$ would be Gaussian with mean:

$$
\mu_{T \mid L_{\top}}=E_{T-1}\left[C_{T}\right]+\Sigma_{22 N}^{-1} \Sigma_{21 N}\left(L_{T}-E_{T-1}\left[L_{T}\right]\right),
$$

and variance

$$
\eta_{T \mid L_{\top}}=\Sigma_{11 N}-\Sigma_{12 N} \Sigma_{22 N}^{-1} \Sigma_{21 N}
$$

Suppose in addition to the observables, one also had realizations of the business cycle latent variable available through time $T$. Using the fact that time 
$T-1$ is a normal expansion phase, we also have the information that

$$
Z_{T}-\theta_{N} Z_{T-1}-\alpha_{N}-\beta_{N L} L_{T-1}=Z_{T}^{*}=\beta_{N C} C_{T}+\sigma(T) \epsilon_{T} .
$$

Using the independence of $\epsilon_{T}$ from innovation to the VAR we obtain an updated normal distribution with mean:

$$
\frac{\frac{\mu_{\mathrm{T} \mid \mathrm{L}_{\mathrm{T}}}}{\eta_{\mathrm{T} \mid \mathrm{L}_{\mathrm{T}}}}+\frac{\beta_{\mathrm{N} C}^{2}}{\sigma^{2}(T)} \frac{Z_{\mathrm{T}}^{*}}{\beta_{\mathrm{N} C}}}{\frac{1}{\eta_{\mathrm{T} \mid \mathrm{L} T}}+\frac{\beta_{\mathrm{N} C}^{2}}{\sigma^{2}(T)}}
$$

and variance

$$
\frac{1}{\frac{1}{\eta_{T \mid L T}}+\frac{\beta_{N}^{2} C}{\sigma^{2}(T)}} .
$$

Clearly if the coincident indicator is tightly related to the value of the business cycle latent variable in the normal growth phase $\left(\beta_{N C}^{2}\right.$ is large relative to $\left.\sigma^{2}(T)\right)$ a large update in the precision and perhaps depending on the size of $Z_{T}^{*}$, the center of the forecast distribution is possible.

There is still more information about $C_{T}$ available in the value of the business cycle latent variable at $T+1$ since this is related to the leading indicator at time $T$. This suggests the state space formulation with measurement equation (assuming for simplicitly period $T$ is a fragile phase):

$$
Z_{T+1}^{*}=\left[\begin{array}{llll}
\beta_{F C} & 0 & 0 & \beta_{F L}
\end{array}\right]\left[\begin{array}{c}
C_{T+1} \\
L_{T+1} \\
C_{T} \\
L_{T}
\end{array}\right]+\sigma(T+1) \epsilon_{T+1},
$$

with $Z_{T+1}^{*}=Z_{T+1}-\alpha_{F}-\theta Z_{T}$. Using this measurement equation along with the transition in equation 2 above and the conditional distribution of $C_{T \mid L_{\top}, Z_{\mathrm{T}}}$, standard filtering and smoothing techniques would produce a distribution for value of the coincident indicator in period $T$ taking into account the values of the current and next period value of the latent variable. Clearly, we would also obtain more precise forecast distributions for the values of the coincident and leading indicators at time $T+1$.

In the general case where the largest horizon of the leading indicator is $L$, if we had the values of the latent variable thru time $T+L$, then we could 
incorporate this information in the forecast distributions for the coincident and leading indicators using similar methods. Suppose we use these forecast distributions to generate realizations of the future values of the leading and coincident indicators. We can use these realizations along with the known set of observable data to generate a new sequence of realizations of the latent variable using techniques discussed below in Section 3.2. Iterating this alternating conditioning a large number of times would generate draws from the unconditional (i.e., not dependent on the latent variable) forecast distribution for future observables, see Section 4.1. We will use this approach in our MCMC algorithm so the relevant estimation period for parameters and latent variables will be $T+L$.

\subsubsection{Parameters For the $\mathrm{N}$ onlinear VAR}

We signify the set of parameters of the nonlinear model by $\Xi$. This set can be decomposed into five subsets $\left\{\Xi_{i}\right\}$ with respective elements:

$$
\begin{gathered}
\boldsymbol{\xi}_{1}=\left[\partial_{1}^{\prime}, \operatorname{vec}\left(\Lambda_{1}\right)^{\prime}, \cdots, \partial_{5}^{\prime}, \operatorname{vec}\left(\Lambda_{5}\right)^{\prime}\right]^{\prime}, \\
\boldsymbol{\xi}_{2}=\left[\operatorname{diag}\left(\Pi_{1}\right)^{\prime}, \cdots, \operatorname{diag}\left(\Pi_{5}\right)^{\prime}\right], \\
\boldsymbol{\xi}_{3}=\left[\operatorname{diag}\left(\Sigma_{1}^{u}\right)^{\prime}, \cdots, \operatorname{diag}\left(\Sigma_{5}^{u}\right)^{\prime}\right], \\
\boldsymbol{\xi}_{4}=\left[\mathbf{b}_{1}^{\prime}, \operatorname{vec}\left(\Theta_{1}\right)^{\prime}, \cdots, \mathbf{b}_{5}^{\prime}, \operatorname{vec}\left(\Theta_{5}\right)^{\prime}\right]^{\prime}
\end{gathered}
$$

and

$$
\boldsymbol{\xi}_{5}=\left[\operatorname{vec}\left(\Sigma_{1}^{\mathrm{W}}\right), \cdots, \operatorname{vec}\left(\Sigma_{5}^{\mathrm{W}}\right)\right]
$$

\section{Defining and I dentifying B usiness Cycle P hase \\ 3.1 Definitions of Business Cycles}


The joint model described above has 5 distinct business cycle phases. We define these phases in terms of the behavior of the latent variable $\left\{Z_{t}\right\}$. We start by defining downturns as periods when $Z_{t}<0$. Next we distinguish recoveries from other periods of expansion by their proximity to periods when $Z_{t}$ changes from negative to positive. That is, $t \in R$ if $Z_{t}>0$ and $\sum_{s=0}^{\infty} \prod_{w=0}^{s} 1\left[Z_{t-w}>0\right]<\varpi$

The other three phases of an expansion are defined to be feasible after $\varpi-1$ periods of recovery. They are defined by a simple partition of the positive reals:

1. $t \in F$ if $0 \leq Z_{t}<r_{1}$ and $\sum_{s=0}^{\infty} \prod_{w=0}^{s} 1\left[Z_{t-w}>0\right] \geq \varpi$,

2. $t \in N$ if $r_{1} \leq Z_{t}<r_{2}$ and $\sum_{s=0}^{\infty} \prod_{w=0}^{s} 1\left[Z_{t-w}>0\right] \geq \varpi$,

3. $t \in S$ if $Z_{t}>r_{2}$ and $\sum_{s=0}^{\infty} \prod_{w=0}^{s} 1\left[Z_{t-w}>0\right] \geq \varpi$.

In order to define the sequence of scalings of the innovations to the latent variable model we need to define a business cycle. We focus on two definitions:

1. A business cycle begins with the start of complete recovery phase, ends the period before a transition from a downturn phase to a recovery phase and must include at least one phase from normal, spurt, fragile. Interrupted recovery phases do not belong to any business cycle.

2. A business cycle begins with the start of a complete recovery phase, ends the period before a transition from a downturn phase to a complete recovery phase and must include at least one phase from the three nonrecovery expansion phases.

Both definitions have difficulty classifying sequences that involve interrupted recoveries. Definition 1 compromises by not classifing all periods as belonging to a particular business cycle. Definition 2 places interrupted recovery phases at the end of a business cycle. In terms of the parametrization of the innovation variance Definition 2 associates with each complete business cycle a different value for the innovation variance. For Definition 1 we can also associate a different value for the innovation variance with each complete business cycle but we also need an innovation variance for interrupted recoveries. One possibility is to allow for unique innovation variances for each 
interrupted recovery, an alternative is to use the same innovation variance for each interrupted recovery.

These assumptions determine a transition function across the various phases. Because the innovation to $Z_{t}$ can vary across business cycles the transition function is not time homogenous. Further, the restriction on the minimum time in the recovery period implies that the transition function is not aperiodic. However, some properties of the transition function are immediate. First, once in a downturn phase the only transition allowed is to a recovery phase. Second, the only transition allowed from complete recovery phases are to expansion phases. Third, we expect the combination of our prior and the data to impose an ordering of how likely downturn phases are such that they are most likely from fragile phases, less likely from normal growth phases and very unlikely from spurt phases. To investigate restrictions required for the latter conjecture define a standardized predicted value for the latent variable by:

$$
\widehat{Z}_{t \mid t-1}=\frac{\widehat{X}_{P t}+\theta_{P} Z_{t-1}}{\sqrt{\sigma^{2}(t)+\boldsymbol{\beta}_{P}^{\prime} \mathrm{H} \Sigma_{P} \mathrm{H}^{\prime} \boldsymbol{\beta}_{P}}},
$$

where $\widehat{X}_{P t}=\alpha_{P}+\boldsymbol{\beta}_{P}^{\prime}\left(\mathrm{a}_{P}+\mathbf{A}_{P} \mathbf{Y}_{t-1}\right)$. The probability that $Z_{t}>0$ is given by the cumulative distribution function of a standard normal evaluated at $-\widehat{Z}_{t \mid t-1}$. An obvious sufficient condition for downturns to be less likely from spurt phases than fragile phases is:

$$
\begin{aligned}
& \left(\widehat{X}_{F t}+\theta_{F} Z_{t-1}\right)\left(\sqrt{\sigma^{2}(t)+\boldsymbol{\beta}_{S}^{\prime} \mathrm{H} \Sigma_{S} \mathrm{H}^{\prime} \boldsymbol{\beta}_{S}}\right) \\
<\quad & \left(\widehat{X}_{S t}+\theta_{S} Z_{t-1}\right)\left(\sqrt{\sigma^{2}(t)+\boldsymbol{\beta}_{F}^{\prime} \mathrm{H} \Sigma_{F} \mathrm{H}^{\prime} \boldsymbol{\beta}_{F}}\right) .
\end{aligned}
$$

In general one would expect $\widehat{X}_{F t}+\theta_{F} Z_{t-1}<<\widehat{X}_{S t}+\theta_{S} Z_{t-1}$ because of the stronger growth and higher level of the latent variable in spurt phases. However, if $\boldsymbol{\beta}_{S}^{\prime} \mathrm{H} \Sigma_{S} \mathrm{H}^{\prime} \boldsymbol{\beta}_{S}>>\boldsymbol{\beta}_{F}^{\prime} \mathrm{H} \Sigma_{F} \mathrm{H}^{\prime} \boldsymbol{\beta}_{F}$ then the inequality might not hold. Requiring that $\Sigma_{F}-\Sigma_{S}$ is a positive definite matrix, i.e., that fragile phases are more uncertain that spurt phases, would remove this possibility if the elements of $\boldsymbol{\beta}_{S}$ and $\boldsymbol{\beta}_{F}$ were identical. Alternatively the inequality would hold if $\boldsymbol{\beta}_{S}$ weighted more heavily variables with low variance and more lightly variables with high variance than $\boldsymbol{\beta}_{F}$.

Now consider the normal phase. We would like to make this the most persistent phase. Once again consider the standardized predicted value. If 
we try to introduce greater persistence directly by imposing $\theta_{N}>\theta_{P}, P \neq N$, then we run into the problem that this also increases the overall variability of $Z_{t}$ which makes it more likely it will cross the thresholds of $r_{1}$ or $r_{2}$. We can counteract this increase in variability by reducing the variance of $\boldsymbol{\beta}_{N}^{\prime} \boldsymbol{Y}_{t} \boldsymbol{\beta}_{N}$ in normal phases assuming that the variability of $\boldsymbol{\beta}_{P}^{\prime} \mathbf{Y}_{t} \boldsymbol{\beta}_{P}$ is a major source of transitions in other phases. The variance of $Y_{t}$ also has two similar sources: $\Sigma_{N}$ and $\mathrm{A}_{N}$. Consider the counterfactual case of remaining in the normal phase forever then the variance would converge to

$$
\left[\mathbf{I}_{M^{2}}-\mathbf{A}_{N} \otimes \mathbf{A}_{N}\right]^{-1} \operatorname{vec}\left(\Sigma_{N}\right),
$$

if the largest eigenvalue of $A_{N}$ was inside the unit circle. If the largest eigenvalue was on or outside the unit circle then from any initial value in the normal phase the variance of $Y_{t}$ will increase. This suggests for the normal phase to be more persistent than other phases of the business cycle we require a combination of less persistence in the dynamics of the observables, less uncertainty in one-step head forecasts and $\boldsymbol{\beta}_{N}$ picking out lower variance elements of $\mathrm{Y}_{t}$.

Now consider the effect of a drop in $\sigma^{2}(t)$ as time goes on. This will have the biggest effect on the persistence of business cycle phase where the the sequence of ratios

$$
\frac{\sigma^{2}(t)}{\boldsymbol{\beta}_{P}^{\prime} \mathrm{H} \Sigma_{P} \mathrm{H}^{\prime} \boldsymbol{\beta}_{P}}, \frac{\sigma^{2}(t+1)}{\boldsymbol{\beta}_{P}^{\prime}\left(\mathrm{H} \Sigma_{P} \mathrm{H}^{\prime}+\mathbf{A}_{P} \mathrm{H} \Sigma_{P} \mathrm{H}^{\prime} \mathrm{A}_{P}^{\prime}\right) \boldsymbol{\beta}_{P}}, \ldots
$$

is the largest. We are assuming this is normal phase. Hence as the transition function of the normal phase exhibits more persistence as $\sigma^{2}(t)$ declines we would observe a drop in the volatility of economic time series.

\subsubsection{Similarities and Contrasts with $\mathrm{M}$ arkov Switching $\mathrm{M}$ odels}

Suppose that $\theta_{P}=0$ and $\boldsymbol{\beta}_{P}$ was a zero vector for all 5 of the phases and set $\sigma(t)=1$. Then transitions between phases would very similar to a Markov Switching model with duration dependence (see Durland and McCurdy 1994). If we removed the restriction on the recovery phase then we would have something very close to Hamilton's original Markov switching model. The main diffference would be the timing convention. In standard Markov switching applications the regime classification variable is contemporaneous in the time series model of interest. In our case the regime classification variable is lagged one period. Under the constant hazard of transition 
assumption this is a minor difference, although below in footnote 2 we suggest how our timing convention would lend itself to the incorporation of information from NBER business cycle dates into the regime classification.

If we allow $\boldsymbol{\beta}_{P}$ to be non-zero vector for each phase then superficially our model is similar to DLW\&F. However, in this case the timing convention produces a significant difference. In the Markov switching approach the elements of $\boldsymbol{\beta}_{P}$ are zero for all coincident variables, and it is assumed that the dynamics of the coincident and leading variables can be modeled separably with the (linear) VAR for leading variables containing no information on regime classification. ${ }^{1}$ The contemporaneous state then determines the regime for the VAR of the coincident variable.

In our approach the coincident variable is used to determine the classification of the business cycle phase which in turn effects the dynamics of the VAR. Markov switching models infer that if the economy is in a recession state then coincident variables should be negative. In our approach if the coincident variable is negative then we infer it is more likely that the economy is a recession phase and the forecast of the coincident and leading variables for next period should be adjusted accordly.

Finally consider allowing for autoregressive dynamics in the latent variable. Without the autoregressive dynamics the latent variable forgets its location within particular phases in the previous period. For example, suppose $Z_{t-1}$ and $t-1 \in F$. Without the autoregressive component the probability of transition to a new phase is the same if $Z_{t-1} \approx 0$ or if $Z_{t-1} \approx r_{1}$. With the autoregressive dynamics the conditional mean is increased by approximately $\theta_{4} r_{1}$. This extra term includes information on the strength of the leading and coincident indicators from previous periods. The standard Markov switching approach ignores this information.

\subsection{M odel based I dentification of B usiness Cycle P hases}

The identification of the business cycle phases has two main sources. First, we can use the model itself and data to classify the phases. Second we can use the dating of business cycles produced by the NBER.

To illustrate the identification from the model assume that all the values of the parameters are known and all values of $Z_{t}$ were known except at time

\footnotetext{
${ }^{1}$ The papers are not explicit on the dynamics of the leading indicators but if we assumed that they have linear dynamics then no information is lost in treating them as exogenous.
} 
$\tau$. This type of conditioning is used extensively in the MCMC algorithm below where repeated interation over parameter draws allows one to make unconditional inferences about the phase classification of a particular period. Consider the case that $r_{1}>Z_{\tau-1}>0, Z_{\tau+1}<0$ and $\sum_{s=1}^{\infty} \prod_{w=1}^{s} 1\left[Z_{\tau-w}>0\right]$ $>\varpi$. Thus, period $\tau$ is any phase except a recovery. There are two sources of information the fit of the nonlinear var for period $\tau+1$ and the latent variable model for periods $\tau, \tau+1$. Using the independence between the innovations to the the nonlinear VAR and the latent variable model we consider the update in two steps. First, we evaluate the height of the likelihood of the nonlinear var for period $\tau+1$ for the four possible phases:

$$
\begin{aligned}
\ell\left(\mathbf{Y}_{\tau+1}, \mathbf{Y}_{\tau} \mid \tau\right. & \in P) \\
& =2 \pi^{-K / 2}\left|\operatorname{det}\left(\Sigma_{P}\right)\right|^{-0.5} \exp \left[-0.5 \mathrm{~V}_{P \tau+1}^{\prime} \Sigma_{P}^{-1} \mathrm{~V}_{P \tau+1}\right], P \neq R
\end{aligned}
$$

Note that given the information that $\tau+1$ is a downturn phase, the two most likely regimes should be fragile or downturn. If $\tau$ is assumed to be a spurt phase then one might expect its predictions of $Y_{\tau+1}$ to be relatively poor. Starting from a prior, $b(\tau \in P)$, over the phases at time $\tau$ we obtain the updated probability mass function:

$$
f\left(\tau \in P \mid \mathbf{Y}_{\tau+1}, \mathbf{Y}_{\tau}\right)=\frac{\ell\left(\mathbf{Y}_{\tau+1}, \mathbf{Y}_{\tau} \mid \tau \in P\right) b(\tau \in P)}{\sum_{P \neq R} \ell\left(\mathbf{Y}_{\tau+1}, \mathbf{Y}_{\tau} \mid \tau \in P\right) b(\tau \in P)}, P \neq R .
$$

Next we focus in the conditional density $f\left(Z_{\tau} \mid Z_{\tau-1}, Z_{\tau+1}\right)$ (we suppress the dependence on $\mathbf{Y}_{\tau+1}, \mathbf{Y}_{\tau}$ ). This is sufficient from the first order Markov assumption that prevails in the absence of recovery periods. This conditional density equals

$$
\frac{f\left(Z_{\tau+1} \mid Z_{\tau}\right) f\left(Z_{\tau} \mid Z_{\tau-1}\right) f\left(Z_{\tau-1}\right)}{f\left(Z_{\tau-1}, Z_{\tau+1}\right)} .
$$

Since $Z_{\tau-1}, Z_{\tau+1}$ are fixed this density is proportional to

$$
f\left(Z_{\tau} \mid Z_{\tau-1}\right) f\left(Z_{\tau+1} \mid Z_{\tau}\right)
$$

For each phase this product of conditional densities is proportional to:

$$
\exp \left(-\frac{\left(Z_{\tau}-\mu\right)^{2}}{2 \sigma^{2}(\tau+1)}\right) \exp \left(-\frac{\theta_{P}^{2}\left(Z_{\tau}-\mu_{P}\right)^{2}}{2 \sigma^{2}(\tau)}\right)
$$


where

$$
\begin{aligned}
\mu & =X_{F \tau}+\theta_{F} Z_{\tau-1}, \\
\mu_{p} & =\frac{Z_{\tau+1}-X_{P \tau}}{\theta_{P}} .
\end{aligned}
$$

We can re-express this product as a normal density for $Z_{\tau}$, with mean (note we impose $\sigma^{2}(\tau)=\sigma^{2}(\tau+1)$ to simplify the notation):

$$
\bar{\mu}_{P}=\frac{\mu+\mu_{P} \theta_{p}^{2}}{1+\theta_{P}^{2}}
$$

and variance

$$
\bar{\sigma}_{P}^{2}=\frac{\sigma^{2}(\tau)}{1+\theta_{P}^{2}}
$$

multipled by the function:

$$
g_{P}(\tau)=\exp \left[-\frac{0.5}{\sigma^{2}(\tau)}\left(\mu^{2}+\mu_{P}^{2} \theta_{P}^{2}-\frac{\left(\mu+\mu_{P} \theta_{P}^{2}\right)^{2}}{1+\theta_{P}^{2}}\right)\right] .
$$

Thus the likelihood of a phase is given by the value $g_{P}(\tau)$ multiplied by integral of the normal density over the relevant region of the phase:

$$
\ell\left(Z_{\tau-1}, Z_{\tau+1} \mid \tau \in P\right)=g_{P}(\tau) \Delta_{P}\left(Z_{\tau-1}, Z_{\tau+1} ; \mathbf{Y}_{\tau+1}, \mathbf{Y}_{\tau}\right)
$$

Once again one would expect that the fragile phase would be the most likely of the possible expansion phases for period $\tau$ because it should be the most capable of predicting the negative value in period $\tau+1$. By similar reasoning the spurt phase should be the least likely. If we simplify to the case of $\theta_{N}=\theta_{S}=\theta_{F}, \alpha_{N}=\alpha_{S}=\alpha_{F}$ then this requires $X_{S \tau}>X_{N \tau}>X_{F \tau}$. Returning to the example of the term spread. Suppose that all 3 contain the term spread and the term spread was inverted $L$ periods before. Then phase with the largest positive weight on the spread would be the most likely to predict the negative value of the latent variable. Thus, this should be the fragile phase. Further, by 'overweighting' the term spread in the fragile phase one would make it a transition type indicator. Similarly by underweighting it in the normal phase one would reduce the variance in this phase and make a transition from a normal to fragile phase more likely before a downturn if the spread is associated with downturns. 
Combining these likelihood values with the updated probabilities from the nonlinear VAR, the conditional probability of phase $P$ at time $\tau$ is given by:

$\operatorname{Pr}\left[\tau \in P \mid \mathbf{Y}_{\tau+1}, \mathbf{Y}_{\tau}, Z_{\tau-1}, Z_{\tau+1}\right]=\frac{\ell\left(Z_{\tau-1}, Z_{\tau+1} \mid \tau \in P\right) f\left(\tau \in P \mid \mathbf{Y}_{\tau+1}, \mathbf{Y}_{\tau}\right)}{\sum_{P \neq R} \ell\left(Z_{\tau-1}, Z_{\tau+1} \mid \tau \in P\right) f\left(\tau \in P \mid \mathbf{Y}_{\tau+1}, \mathbf{Y}_{\tau}\right)}$

A more difficult case occurs when we need to classify a time $\tau$ that is in the middle of a recovery period. The classification is limited to a downturn or recovery phase. However, selecting a downturn phase has implications for the $\varpi$ periods following $\tau$. At the most extreme suppose that if $Z_{\tau}$ was positive then it would mark the last period of a recovery. Thus, a negative value for $Z_{\tau}$ if $\left\{Z_{\tau+s}>0, s=1, \ldots, \varpi\right\}$ would re-classify $\varpi$ future periods as recovery ones. Since the values of $\left\{Z_{\tau+s}, s=1, \ldots, \varpi\right\}$ are being conditioned on this would introduce the likelihood effect of classifying these $\varpi$ periods as normal, spurt or fragile versus recovery periods. Further, the value of the innovation variance of the latent variable model depends on whether we have a complete recovery has occurred or not.

\subsection{Incorporating NBER Business Cycle Dates}

An additional source of information on business cycle phases is available from the NBER's classification of recessions and expansions. The NBER defines a business cycle as starting the month after a peak and ending the month of the next peak or starting the month after a trough and ending the month of the next trough. Further, they assume that the economy is either in recession, expansion (depression phases are also included prior to 1945) and that expansion phases must last at least 12 months and recession phases must last at least 6 months. The NBER looks at levels of activity to define peaks and troughs, whereas we focus on growth rates of activity measures.

If we assume that NBER defined recessions are identical to downturns as measured by our business cycle latent variable then their business cycle chronology identifies recovery and downturn phases(with the exception of peak and trough months) exactly and by default all other time periods must be classified in one of the three other phases. Further, we do not have the possibility of interrupted recovery.

An interpretation of the exact correspondence between the NBER recessions and downturns is an informative prior over the timing of business cycle 
phases that is degenerate for certain dates. More formally, we are assuming that the NBER dating committee does not observe the same data we are using but instead is able to observe the sign of $Z_{t}$. In the case of Hamilton's original Markov switching model such an assumption would make little sense. Given the "prior information" of the NBER dates the transition matrix parameters could be updated immediately without analyzing the observables by using the length of expansions and recessions excluding peak and trough months. These probabilities would then be slightly updated by using the behavior of the observables at peak and trough months to classify them as recession or expansion months.

Consider the example of generating a value of $Z_{\tau}$ above if the NBER chronology stated that $\tau$ was a recession period. There would be no updating required on the business cycle phase and the missing (negative) value of $Z_{\tau}$ could be generated by drawing $\epsilon_{\tau}$ from the truncated normal on $\left(-\infty,-\bar{\mu}_{D} / \bar{\sigma}_{D}\right)$ (in this case the draw is very similar to Dueker 2001).

We could weaken this assignment of probabilities to allow for some ambiguity. For example, recessions start some time in a peak month and end some time during the trough month according to comments from the NBER dating committee. Thus, suppose the NBER classifies period $\tau$ as a peak month we could assign prior weight 0.5 on a downturn and 0.5 on an expansion. If we distributed the rest of the probability equally amongst the 3 alternatives, this would make it 3 times more likely ex-ante that $\tau$ was a downturn than a fragile period. It makes more sense to give the fragile phase the same ex-ante weight thus limiting the classification to 2 phases.

There is less of an issue with assigning probabilities for trough months since by our definition of recovery phases, all other expansion phases are ruled out. The NBER requirement that expansions last at least 12 months implies that in monthly data the maximum a priori value of $\varpi$ is less than 12. (If we allow it to be larger, then the number of business cycles would not agree).

One could use the information in the NBER dates in further ways. For example, in the periods before a business cycle peak one could place a high prior weight on a fragile phase. If one wanted to be design a model that was good at predicting NBER peak dates one could also place low prior weight on a fragile phase in expansion periods not close to a business cycle peak (see Leamer 2001 for a related approach).

Alternatively, one could examine a prior over business cycle phases that was between the uniform one and NBER prior described above. For exam- 
ple, the shortest expansion in the NBER chronology is August 1980 to July 1981 which lasted only the minimum 12 months. We could choose to view this period as an interrupted recovery following by a sustained downturn by relaxing the prior that it was a complete recovery. ${ }^{2}$

Below if we condition on the exact NBER dates (with peak and trough periods treated as above) we use the symbol $\mathcal{N}$, if we use the dates but do not impose exact correspondence between the NBER recessions and our downturn phase we use the symbol $\mathcal{M}$.

\section{Forecasting}

\subsection{Simulating $\mathrm{T}$ he $\mathrm{J}$ oint $\mathrm{M}$ odel and Forecasting}

It is useful to write the dynamics of the latent variables in a companion form by defining the vector:

$$
\mathbf{Z}_{t}=\left[\begin{array}{c}
Z_{t} \\
Z_{t-1} \\
Z_{t-\varpi-1}
\end{array}\right]
$$

Thus, we can describe the dynamics of the system over business cycle $j$ by the following transition function

$$
f_{j}\left(\mathbf{Y}_{t}, \mathbf{Z}_{t} \mid \mathbf{Y}_{t-1}, \mathbf{Z}_{t-1}\right)=f_{j}\left(\mathbf{Z}_{t} \mid \mathbf{Y}_{t}, \mathbf{Z}_{t-1}\right) f\left(\mathbf{Y}_{t} \mid \mathbf{Z}_{t-1}, \mathbf{Y}_{t-1}\right)
$$

Here $f_{j}\left(\mathbf{Z}_{t} \mid \mathbf{Y}_{t}, \mathbf{Z}_{t-1}\right)$ and $f\left(\mathbf{Y}_{t} \mid \mathbf{Z}_{t-1}, \mathbf{Y}_{t-1}\right)$ are conditionally Gaussian.

In order to simulate the dynamics of this transition function we need to follow a number of steps.

1. Draw a realization of $\mathbf{Y}_{t+1}$ from $f\left(\mathbf{Y}_{t+1} \mid \mathbf{Z}_{t}, \mathbf{Y}_{t}\right)$.

2. Draw a realization of $Z_{t+1}$ from $f_{j}\left(Z_{t+1} \mid Z_{t}, Y_{t+1}\right)$.

3. Using these two realized values draw $\mathrm{Y}_{t+2}$ from $f\left(\mathrm{Y}_{t+2} \mid \mathrm{Z}_{t+1}, \mathrm{Y}_{t+1}\right)$.

\footnotetext{
${ }^{2}$ One could apply a similar principle to the estimation of Hamilton's original Markov switching model by placing a non-degenerate but NBER informed prior on the whether a particular time period is a recession or expansion period. This prior would induce an informative prior on the transition matrix probabilities that would then be updated using the behavior of observables.
} 
4. Using the draws of $Z_{t+1}$ and $Y_{t+2}$ draw $Z_{t+2}$ from $f_{j}\left(Z_{t+2} \mid Z_{t+1}, Y_{t+2}\right)$.

5. Continue this process until we have simulated to the desired horizon, note that if the horizon includes the end of one complete business cycle then the conditional distribution for the latent variable could change and we need to amend the simulation as described below.

Repeating this process a large number of times would produce draws from the (conditional on $Z_{t}, Y_{t}$ ) marginal distributions of $Z_{t+h}$ and $Y_{t+h}$. Generating draws for $\mathbf{Y}_{t+h}$ conditional only on $\mathbf{Y}_{t}$ is more difficult. The MCMC algorithm will generate draws of the latent variable conditional on the whole sample. That is, the actual values of $\left\{Y_{t}\right\}$ were used. In Markov switching models one can use the filter probabilities that only depend on the past observed values to integrate out the latent variable. In our case the generated values after time $T$ depends only on past values of the observables. The sampler automatically generates draws thru period $T+L$. Averaging these forecasts across iterations of the sampler would produce a forecast conditioned only on observables. Further one can generate forecasts with origin $T+L$ at each iteration of the MCMC sampler using the steps described above to generate draws from the forecast distribution.

\subsection{Predicting R ecovery $P$ hases}

We have allowed the innovation variance to the latent variable model to depend on the business cycle number. This means that if a new business cycle is forecast to begin we need to use a new value for the variance. Under both definitions we only know if a new business cycle has begun if the expansion lasts for $\varpi$ periods. Thus, once in a downturn we would need to evaluate the probability of a consecutive sequence of $\varpi$ positive values for the latent variable using the new innovation variance and probability of all other sequences using the old innovation sequence or the special interrupted recovery value.

If we do have a complete recovery sequence then we know that the nonlinear VAR will be in the recovery phase for next $\varpi-1$ periods with the initial phase being a downturn. Thus, we can act as if its forecast distributions are Gaussian. A similar argument works for the latent variable model. We can use this multivariate Gaussian distribution to evaluate the probability of $\varpi$ positive values of the latent variable. For the case of an interrupted recovery we form a similar Gaussian distribution but using $\sigma^{2}(J)$. We are interested 
in the complement to a consecutive sequence of $\varpi$ positive values for the latent variable so we can calculate the same type of probability.

If a complete recovery phase is chosen then we forecast as described above with the restriction that only positive values of the latent variable are allowed for $\varpi$ periods. If an interrupted recovery or continuing downturn is chosen then we can simulate period by period as follows (using either the old innovation sequence or where appropriate the interrupted recovery value):

1. If $Z_{T+l+1}<0$, then we need to reevaluate the probability of a complete recovery with the new realizations.

2. If $Z_{T+l+1}>0$ then simulate as above.

3. Continue period by period as in (1) and (2) above, until

4. If no negative values are realized by $T+h+\varpi-1$ then $Z_{T+l+\varpi}$ must be negative.

\subsection{Example:P redicting R ecessions/ Downturns}

Here we contrast our model with others in the literature where the goal is to predict recessions. Estrella and Mishkin (1998 and references herein, EM hereafter) discuss using probit models to predict NBER business cycle classifications of expansions and recessions. In their approach there is also implicitly a latent variable $Z_{t}$ that takes on positive values in expansions and negative values in recessions:

$$
Z_{t}=\mathbf{X}_{t}+\epsilon_{t}
$$

where the vector $\mathbf{X}_{t}=\alpha+\boldsymbol{\beta} \mathbf{Y}_{t}$. In the most frequent application the elements of the vector $\boldsymbol{\beta}$ are zero except for one lag of the term spread. EM use the observed NBER business cycle dates to classify when the latent variable is positive (expansions) and negative (recessions). ${ }^{3}$ EM find that using additional leading indicators to the yield curve does not help in real time prediction because of overfitting in sample. The main empirical finding of this literature is that inversions of the yield curve lead recessions. Thus, the probability of a recession state a time $t$ is given by $\Phi\left(-\mathbf{X}_{t}\right)$.

\footnotetext{
${ }^{3} \mathrm{EM}$ us the convention that the peak month is part of an expansion and the trough month is part of a recession.
} 
Assume that we use a similar lead for the yield curve as EM but also include a coincident variable such as employment. Assume also that we use the available NBER dates in the estimation period. In the EM approach there is no lagged value of the latent variable so the current value (and sign) of the latent variable is not used in forecasting. In our case we require both the current value of the latent variable both because it is useful in predicting the latent variable and because it defines the phase of the business cycle.

Assuming that realizations of the lagged latent variable and coincident variable are available (but the current value of the leading indicator is not yet available), the probability of a negative value of $Z_{t}$ is

$$
\operatorname{Pr}\left[Z_{t}<0 \mid Z_{t-1}, Y^{t-1}, t-1 \in P, \mathcal{N}_{t-1}\right]=\Phi\left[-\frac{\widehat{X}_{P t}+\theta_{P} Z_{t-1}}{\sqrt{\sigma^{2}(t)+\beta_{P C}^{2} \sigma_{P C}^{2}}}\right]
$$

where $\widehat{X}_{P t}=\alpha_{P}+\beta_{P L} L_{t-1}+\beta_{P C} E_{t-1}\left[C_{t}\right]$ and $\sigma_{P C}^{2}=E_{t-1}\left[\left(C_{t}-E_{t-1}\left[C_{t}\right]\right)^{2}\right]$. If we average this probability over the unknown latent variable and business cycle phase we would obtain

$$
\operatorname{Pr}\left[Z_{t}<0 \mid Y^{t-1}, \mathcal{N}_{t-1}\right] .
$$

However, this probability does not answer the question of whether a recession started at time $t$ since one of the phases we averaged over is the downturn phase. To address this issue, consider the first hitting time given by

$$
\mathcal{H}_{D}(t)=\{\mathcal{H}: t+\mathcal{H} \in D, s \notin D, t<s<t+\mathcal{H}\},
$$

with associated probability

$$
\pi_{\ell}(t)=P\left[\mathcal{H}_{D}(t)=\ell\right]=P\left[Z_{t+\ell}<0 \mid Z_{t+\ell-1}>0, \ldots, Z_{t+1}>0\right]\left(1-\pi_{\ell-1}(t)\right) .
$$

For each initial period $t$ we can form an expected hitting time:

$$
\mathbb{E}\left[\mathcal{H}_{D}(t)\right]=\sum_{\ell=1}^{\infty} \ell \pi_{\ell}(t) .
$$

Returning to the EM probit approach. The probability of a recession state a time $t$ is given by $\Phi\left(-\mathbf{X}_{t}\right)$ and conditional on $\mathbf{X}_{t}$ is independent of the probability of a recession state at time $t^{\prime}$. 
In the model of EM assume we have available the conditioning sequence $X_{t-\ell}^{t}=\left\{X_{t}, X_{t-1}, \ldots, X_{t-s}\right\}$ where $t-s$ is the last period in which we are sure the economy was expanding. Thus we can evaluate the probability of first hitting time to recession by:

$$
\pi\left(t-s, X_{t-s}^{t}, \mathcal{N}_{t-1}\right)=\Phi\left(-X_{t}\right) \prod_{\ell=1}^{s}\left[1-\Phi\left(-X_{t-\ell}\right)\right]
$$

Note that this expression reflects a conditionally constant probability of recession and is strictly declining in $\ell$.

The first hitting time probability for our model given $Y^{t-1}$ and $Z_{t-s}^{t-1}>0$,

$$
\begin{aligned}
\pi\left(t-s, \mathbf{Y}^{t-1}, Z_{t-s}^{t-1}>\right. & \left.0, \mathcal{N}_{t-1}\right)=\Phi\left[-\frac{\widehat{X}_{P t}+\theta_{P} Z_{t-1}}{\sqrt{\sigma^{2}(t)+\beta_{P C}^{2} \sigma_{P C}^{2}}}\right] \\
& \prod_{\ell=1}^{s}\left(1-\Phi\left[-\frac{X_{P t-\ell}+\theta_{P} Z_{t-\ell-1}}{\sigma(t-\ell)}\right]\right) .
\end{aligned}
$$

Once again we need to integrate over the unobserved lagged latent variable but with the additional restriction that the values are all positive, i.e. they belong to one of three non-recovery expansion phases.

\section{Estimation and Prior Distribution}

\subsection{Outline of Gibbs Sampler}

Despite its complexity the model is relatively easy to estimate using a Gibbs Sampler with data augmentation. One obvious advantage of this approach is that it generates realizations of the unobserved process $\left\{Z_{t}\right\}$. Given these sequences estimation of the remaining parameters is relatively simple. The sampler is split into 5 main blocks: given the parameters of the latent variable model, $\Psi$, the parameters of the nonlinear VAR, $\Xi$, the (augmented) data $Y^{T+L}$, information from the NBER turning points, the generation of $\left\{Z_{t}\right\}$. Given this sequence, the generation of the latent model parameters. Given the sequence of $\left\{Z_{t}\right\}$ and the latent variable parameters, and the (augmented) data $Y^{T+L}$, the generation of the VAR parameters, $\Xi$. Fourth, given the sequence of $\left\{Z_{t}\right\}$, the business cycle latent variable parameters, and the VAR 
parameters, $\Xi$ the generation of the common factors and the (augmented) data $Y^{T+L}$. Fifth given the sequence of $\left\{Z_{t}\right\}$, the (augmented) data $Y^{T+L}$, the VAR parameters, $\Xi$, the generation of the business cycle classification parameters $r_{1}, r_{2}$ and $\varpi$. Blocks 1 and 4 of the sampler produces forecasts of out of sample values, estimates of data not yet released and probabilistic business cycle classifications. The specific details of the Gibbs Sampler are given in the appendix.

\subsection{Priors}

A major advantage of the Bayesian approach, in addition to its feasibility, is that it allows us to incorporate various forms of prior information into the analysis. We discuss this in general terms below and specifically below in the context of two empirical applications. The model as set out above has a large number of parameters. If we don't restrict these parameters it is unlikely that the output of such a complex model will be sensible. Thus, we focus on ways to impose our prior belief that very few of these parameters should be important in determining the dynamics but still allowing flexibility in response to the data.

\subsubsection{Priors On Latent Variable M odel Parameters}

The prior information on the latent variable parameters comes in a number of forms. Firstly, we have the classification of economic variables into leading and coincident. This gives us information on the pattern of likely non-zero elements in $\boldsymbol{\beta}_{P}$ vectors. Further, we expect that the derived common factors are more likely to have non-zero weights than the individual variables.

For the common factor variables we use a shrinkage type prior designed to allow leading indicators to ahve predictive power at longer leads and coincident variables to have most of their predictive power contemporaneously or with one lag.

For remaining more doubtful elements we use a variable selection prior (similar to George and McCulloch) with an underlying t distribution for the individual coefficients.

In terms of the autoregressive dynamics we impose stationarity and positive persistence. 


\subsubsection{Priors on the Nonlinear Var Parameters}

As the number of variables $(K)$ grows the parameters required for the VAR model starts to become very large without the common factor structure: $5\left(K^{2}(q+1)+K(K+1) / 2\right)$. For example, with $K=12, q=12,9750$ parameters would be required compared to a likely sample of 600 observations on the 12 variables. If we limit ourselves to 2 common factors and restrict the only changes across phases to the common factors, then the number of parameters to be estimated is $5\left(2^{2}(q+1)+2(2+1) / 2\right)+k(3 q+2)$. Again with $k=10, q=12,435$ parameters would be required to be estimated (assuming no lags in the factor loading matrix, 775 parameters with 12 lags in the factor loading matrix). There would be 55 different parameters in each phase for the common factor model. These numbers could be reduced by assuming commonality across expansion phases in the parameters.

A priori it is unlikely that all the parameters in the VAR for the common factors are non-zero thus we use informative shrinkage priors in the form of Litterman et al. based on a classification of the factors into leading and coincident.

\section{A pplication One: U nemployment and Term Spread}

We start by considering a small scale application to one coincident variable, the unemployment rate and one leading variable, the spread between the Fed Funds Rate and the 10 year Treasury Note. These variables were chosen because they have been successful in either predicting or classifying business cycles in a number of previous studies. In Leamer (2001) the spurt regime was identified by late expansion declines in the unemployment rate with knowledge of a surge in real activity. These late expansion declines in the unemployment rate tend to occur with both low and high spreads between short and long term interest rates. On the other hand, the spread tends to be high in early parts of long expansions. Thus we assume a priori that the spread coefficient is relatively small in the normal and spurt phases. We also use the NBER dates as an informative and sometimes degenerate prior as described above in section 3.3 through September 2001. For the period after September 2001 we use a prior that corresponds to a median recession length of 11 months. 
The spread variable is available starting from July 1954 through April 2002. We infer one earlier value of the spread variable for June 1954, hence the relevant estimation sample starts in June 1955. The augmented data set goes up to April 2003 and automatically provides predictive distributions over unemployment, the spread and business cycle phases.

The results from a sampler run of 30,000 with a burn-in phase of 10,000 iterations are shown in Figures 1 to 7 . Figure 1 shows the posterior mean of business cycle phases using the rule $D=1, R=2, N=3, F=4, S=5$. It is clear that the August 1980 to July 1981 expansion is treated as an interrupted recovery period. Figure 2 plots the posterior mean probabilities of the fragile and spurt phases along with the downturn phase (NBER recessions). Somewhat surprisingly the spurt phase only occurs in the mid part of the 1990s expansion and appears to be present in the late 1970s expansion.

Figures 3 and 4 contain information of $\boldsymbol{\beta}_{P}$ for the spread and unemployment rate respectively. The difference between the leading and coincident indicator designation is obvious from the reverse pattern of the coefficients against the lead. Notice that the spread is a particular useful predictor of the fragile phase. The unemployment coefficients appear to suggest that it is changes in the unemployment rate that are important. The spread also has an ambiguous effect in recessions.

Figure 5 contains a plot of the posterior probability that a new business cycle has started (i.e., the recession is over). The probabilities are surprisingly

low. From Figure 3 we can see that the spread and the unemployment rate have little explanatory power for recoveries.

Figures 6 and 7 contain predictive distributions for the values of the Spread and the Unemployment rate in April 2003 generated by the Gibbs sampler.

\section{A pplication Two: Conference Board Lead- ing and Coincident Indicators}

We will use the 10 leading indicators of Conference Board and the 4 coincident indicators used by the Conference Board and in a closely related form by the NBER and Stock and Watson. One version will assume that the common factors are the composite indicators produced by the Conference Board, another will estimate a leading and coincident common factor using 
the Gibbs sampler.

\section{Conclusion}

\section{A ppendix: Description of the Gibbs Sampler}

\section{Business Cycle Classification Variable}

Conditional on values for latent model parameters $\Psi$, the VAR parameters, the augmented data and the business cycle classification parameters we want to combine the prior information on $\left\{Z_{t}\right\}$ generated by the NBER classification system with the behavior of the observable economic time series. As described in the main text in Section 3.2 this can be accomplished by conditioning on the value of $Z_{t-1}$ from the previous iteration of the sampler and $Z_{t+1}$ from the current run of the sampler (See Albert and Chib (1993) for the original application of this approach).

The Gibbs sampler is then implemented exactly as described in Section 3.2 with the exception of the first and last observation. In the case of the last observation we only condition on $Z_{T+L-1}$ and there is no information available from the nonlinear VAR. Thus, this is a simple Gausian draw using the relevant phase from Equation 1. For the first observation we condition on $Z_{2}$ and combine the information from the VAR with that from the latent variable model in a similar manner to Section 3.2 for the case where the mean of $Z_{1}$ is $\mu_{P}$ and its variance is $\sigma^{2}(1) / \theta_{P}^{2}$.

\section{Latent Model Parameter Draws}

Given the draw of the latent variable the conditional mean and variance of the parameters of the threshold model in Equation 1 can be found in a number of ways. We focus on blocking $\Psi$ into the four sets described above in Section 2.1.

\section{Leading and Coincident Parameters}

First, conditional on the autoregressive structure, $\psi_{2}$ and business cycle specific variances, $\psi_{3}$ and using a Gaussian prior on $\psi_{1}$ with mean $\mu\left(\underline{\psi}_{1} ; \varsigma\right)$ and 
variance $\Omega\left(\underline{\psi}_{1} ; \varsigma\right)$ we have a Gaussian posterior with variance

$$
\Omega\left(\bar{\psi}_{1} ; \varsigma\right)=\left[\Omega\left(\underline{\psi}_{1} ; \varsigma\right)^{-1}+\mathbf{X}^{\prime} \mathbf{X}\right]^{-1}
$$

and mean

$$
\mu\left(\bar{\psi}_{1} ; \varsigma\right)=\Omega\left(\bar{\psi}_{1} ; \varsigma\right)\left[\Omega\left(\underline{\psi}_{1} ; \varsigma\right)^{-1} \mu\left(\underline{\psi}_{1} ; \varsigma\right)+\mathbf{X}^{\prime} \mathbf{Z}^{*}\right]
$$

where

$$
\begin{aligned}
\mathbf{X}=\left[\begin{array}{c}
\mathbf{X}_{2} \\
\vdots \\
\mathbf{X}_{T}
\end{array}\right], \\
\mathbf{X}_{t}=\left[1(t \in D) \mathbf{Y}_{t}^{\prime} 1(t \in R) \mathbf{Y}_{t}^{\prime} 1(t \in N) \mathbf{Y}_{t}^{\prime} 1(t \in F) \mathbf{Y}_{t}^{\prime} 1(t \in S) \mathbf{Y}_{t}^{\prime}\right], \\
\mathbf{z}^{*}=\left[\begin{array}{c}
\sum_{P=1}^{5} 1(t \in P)\left(Z_{2} / \sigma(2)-\theta_{P} Z_{1} / \sigma(1)\right) \\
\vdots \\
\sum_{P=1}^{5} 1(t \in P)\left(Z_{T} / \sigma(T)-\theta_{P} Z_{T-1} / \sigma(T-1)\right)
\end{array}\right] .
\end{aligned}
$$

As discussed in the main text we focus on two ways of parametrizing the prior distribution. The simplest one is a shrinkage prior tailed for the properties of the variable in question, i.e., leading versus coincident, real vs. financial.

The alternative is the variable selection type prior. This requires some extra steps in the Gibbs Sampler using the information in the drawn values of $\left\{\alpha_{P}, \boldsymbol{\beta}_{P}\right\}$. Define the binary random vector $\boldsymbol{\xi}$. Let $\varkappa_{1}$ be the precision associated with inclusion and $\varkappa_{1}$ the variance associated with effective exclusion. Then the probability that an element of $\boldsymbol{\xi}$ is equal to 1 is given by:

$$
\frac{\sqrt{\varkappa_{1}} \exp \left(-0.5 \varkappa_{1} \beta^{2}\right) q}{\sqrt{\varkappa_{1}} \exp \left(-0.5 \varkappa_{1} \beta^{2}\right) q+\sqrt{\varkappa_{2}} \exp \left(-0.5 \varkappa_{2} \beta^{2}\right)(1-q)} .
$$

In the case where the unconditional prior distribution is in the $t$ distribution family there is one further step to find the collection of precisions. Following Geweke (1992) we have:... 


\section{A utoregressive Parameters}

Next conditional on new draw of the parameters, $\psi_{1}$ and business cycle specific variances $\psi_{3}$ the autoregressive parameters are assumed to have a (truncated Gaussian prior with parameters $\mu\left(\underline{\psi}_{2}\right)$ and variance $\Omega\left(\underline{\psi}_{2}\right)$. Ignoring the truncation we have a Gaussian posterior with variance

$$
\Omega\left(\bar{\psi}_{1}\right)=\left[\Omega\left(\underline{\psi}_{1}\right)^{-1}+\mathbf{Z}^{\prime} \mathbf{Z}\right]^{-1}
$$

and mean

$$
\mu\left(\bar{\psi}_{1}\right)=\Omega\left(\bar{\psi}_{1}\right)\left[\Omega\left(\underline{\psi}_{1}\right)^{-1} \mu\left(\underline{\psi}_{1}\right)+\mathbf{Z}^{\prime} \mathbf{Z}\right]
$$

where

$$
\mathbf{Z}=\left[\begin{array}{c}
\mathrm{Z}_{1} \\
\vdots \\
\mathrm{Z}_{T}^{\prime}
\end{array}\right]
$$

$\mathbf{Z}_{t}=\left[1(t \in D) Z_{t} / \sigma(t) \quad 1(t \in R) Z_{t} / \sigma(t) \quad 1(t \in N) Z_{t} / \sigma(t) \quad 1(t \in F) Z_{t} / \sigma(t) \quad 1(t \in S) Z_{t} / \sigma(t)\right]$

$$
\mathbf{z}=\left[\begin{array}{c}
\left(Z_{2}-\sum_{P=1}^{5} 1(2 \in P) X_{P 2}\right) / \sigma(2) \\
\vdots \\
\left(Z_{T}-\sum_{P=1}^{5} 1(T \in P) X_{P T}\right) / \sigma(T)
\end{array}\right]
$$

\section{Business Cycle Specific Innovation Scalings}

Next given these new draws of $\psi_{1}, \psi_{2}$ we construct the squared errors and length for of each business cycle to updated on the innovation scalings.

$$
v s^{2}(j)=\sum_{t \in B_{\mathrm{j}}} \epsilon_{t}^{2}, v_{E}(j)=\sum_{t \in B_{\mathrm{j}}} 1\left(t \in B_{j}\right), \quad j=2, \ldots, J+1 .
$$

We use a standard inverted Gamma prior with degrees of freedom $\underline{v}$,mean scale $\underline{v s}{ }^{2}$. Thus for each business cycle $j<J+1$ we have a Gamma distribution with posterior degrees of freedom

$$
\bar{v}(j)=\underline{v}+v(j)
$$

and posterior mean scale:

$$
\overline{v s}^{2}(j)=\underline{v s}^{2}+v s^{2}(j) .
$$

Draw for New business cycle to be completed 


\section{Nonlinear VAR Parameter Draws}

Given the draw of the latent variables $\left(Z_{t}\right.$ and $\left.\mathrm{cf}_{t}\right)$ and business cycle classification parameters parameters, the nonlinear var parameters can be found using standard techniques for linear models by blocking within this block. We start by defining the column vector $\mathbf{w}=\left[1, \mathrm{cf}_{t-1}^{\prime}, \cdots, \mathrm{cf}_{t-q}^{\prime}\right]$ and the matrix $\mathrm{W}_{P}$ by

$$
\left[\begin{array}{c}
1(1 \in P) \mathbf{w}_{1} \\
\vdots \\
1(1 \in P) \mathbf{w}_{T+L-1}
\end{array}\right]
$$

and

$$
\mathbf{U}=\left[\begin{array}{cccc}
c f_{12} & c f_{22} & \cdots & c f_{s 2} \\
c f_{12} & c f_{22} & \cdots & c f_{s 2} \\
\vdots & \vdots & \vdots & \vdots \\
c f_{1 T+L} & c f_{2 T+L} & \cdots & c f_{s T+L}
\end{array}\right]
$$

We have the least squares estimator (see Lutkepohl 1991)

$$
\widehat{\boldsymbol{\xi}}_{4}=\left[\left(\mathbf{W}_{P}^{\prime} \mathbf{W}_{P}\right) \otimes \mathbf{I}_{s}\right]^{-1}\left[\mathbf{W}_{P}^{\prime} \otimes \mathbf{I}_{s}\right] \operatorname{vec}\left(\mathbf{U}^{\prime}\right)
$$

This can be combined with a Gaussian prior to obtain a Gaussian posterior distribution.

\section{Data A ugmentation Step}

There are two main types of data that we need to augment the algorithm with. The first are the values of the common factors. The second comes from the fact that, as described in the discussion of Subsection 2.4.1, the information in the current value of the leading indicators is useful for updating on the out-of-sample values of the coincident and leading indicators. Further, with a realization of the common factors available it is possible to augment any variables with missing observations at the start of the sample or with differeing frequencies than used to define business cycle phases. We start by describing the draw of the common factors. 


\section{Draw of Common Factors}

There are two sources of information on the common factors: the observed economic time series (augmented to fill out the sample to $T+L$ ) and the business cycle latent variable.

\section{Adding $\mathrm{M}$ issing or $\mathrm{N}$ ew data}

\section{Business Cycle Classification Parameters}

The final block of the algorithm generates the parameters $\varpi, r_{1}, r_{2}$. Information on these parameters is available from both the VAR and business cycle latent variable model. The threshold values are easiest to describe. Among the 3 phases of normal, fragile and spurt varying the values of $r_{1}$ will move observations between the fragile and normal regimes and varying $r_{2}$ will move observations (including the latent ones) between the normal and spurt regimes.

We define a prior in terms of the minimum and maximum percentage of expansion observations that can be either of the fragile or spurt regimes. Given the draw of the latent variable we then assume a uniform prior between the realized values corresponding to these minimum and maximum percentages. The likelihood will be flat as we move in the prior space without changing the classification of phases. At realized data points of the business cycle latent variable the likelihood will move discontinuously. Thus, we find the likelihoods at all of these "jump" points and then weight by the inverse width of the interval between jumps. This gives us a joint posterior over the thresholds which can be drawn from using standard inversion techniques.

For the minimum length of a recovery period, we know that varying the parameter in addition to changing the phase classification can also change the innovation variance to the business cycle latent variable model. Again we assume that the prior disribution is uniform (discrete) between a minimum and maximum value. Once again we enumerate the likelihood values associated with all the a priori values of $\varpi$ and use standard inversion techniques to generate a new draw.

\section{Evaluating the Model}

The model developed is very complicated and it is important to be sure that it adds useful information to the study of business cycles. We propose 
using two main methods to measure the value added of this complexity: real time forecasting and Bayes Factors. Real time forecasting is discussed in the main text and we can evaluate the forecasts of the model for business cycle turning points and important real and financial variables using a number of forecasting criteria against standard models. In this subsection of the appendix we focus on the use of Bayes factors, that is the ratio of marginal likelihoods of the our complex model to the simpler models nested within it. We think the use of Bayes factor is important since they automatically penalize more complicated models.

There are two main dimensions of complexity we examine:

1. Is a simple constant hazard model be sufficient to explain the transition between phases?

2. Is a linear VAR (possibly with phase dependent innovation variance) adequate to explain the observed time series?

In order to calculate the Bayes factors we make extensive use of the Savage-Dickey Density ratio. For nested models the Bayes factor is given by the ratio of the height of the posterior density for the larger model evaluated at the restricted parameter values of the nested model to the height of the prior density for the larger model at the restricted parameters values. In our case we exploit this result in an indirect manner as in Chauvet and Potter (2001). 


\section{R eferences}

[1] Bernanke, B.S. Boivin and Eliasz (2002), "Measuring the effects of Monetary Policy: A factor augmented vector autoregression approach," mimeo Department of Economics, University of Princeton..

[2] Beuadry and Koop (1993) J ournal of Monetary Economics

[3] Carter, C and P. Kohn (1994), "On Gibbs Sampling for State Space Models," Biometrika, 81, 541-553.

[4] Chauvet, M. (1998), "," International E conomic Review

[5] Chauvet and Potter (2001).

[6] Chib, S. (1995), "Marginal likelihood from Gibbs Output," J ournal of A merican Statistical Association, 90, 1313-1321.

[7] Diebold, F. and G. Rudebusch (1992), "Have Postwar Economic Fluctuations been stabilized?, A merican Economic Review, 82(4), 993-1005.

[8] Diebold, F., Lee and Weinbach (1994)

[9] Diebold, F. and G. Rudebusch (1996), "Measuruing Business Cycles: A Modern Perspective," Review of E conomics and Statistics

[10] Diebold, F. and G. Rudebusch (2001) San Francisco Fed

[12] Dueker, M.J. (2001), "," Federal Reserve Bank of St. Louis.

[13] Durland and McCurdy 1994 J ournal of Business and Economic Statistics

[14] Estrella, A. and G. Hardouvelis (1991), "The Term Structure as a Predictor of Real Economic Activity," J ournal of Finance, 46 (2), 555-576.

[15] Estrella, A. and F. S. Mishkin (1998a), "Predicting U.S. Recessions: Financial Variables as Leading Indicators," The Review of Economics and Statistics, 80, 45-61. 
[16] Estrella, A., A.P. Rodrigues, and S. Schich (2000), "How Stable Is the Predictive Power of the Yield Curve? Evidence from Germany and the United States," mimeo, Federal Reserve Bank of New York.

[17] Filardo, A (1994) J ournal of Business and E conomic Statistics

[18] Friedman, B.M. and K.N. Kuttner (1993a), "Why Does the Paper-Bill Spread Predict Real Economic Activity?" in J.H. Stock and M.W. Watson (eds), Business Cycles,

Indicators, and Forecasting. Chicago: University of Chicago Press.

[19] Friedman, B.M. and K.N. Kuttner (1998), "Indicator Properties of the Paper-Bill Spread: Lessons from Recent Experience," The Review of Economics and Statistics, 80, 34-44.

[20] Gertler, M. and C.S. Lown, "The Information in the High Yield Bond Spread for the Business Cycle: Evidence and Some Implications," NBER Working Paper No. 7549.

[21] Hamilton (1989) E conometrica

[22] Kim, C-J. and C. Nelson (1999)

[23] Kim, C-J. and C. Nelson (1999), "Has the U.S. Economy Become More Stable? A Bayesian Approach Based on a Markov Switching Model of the Business Cycle," Review of Economics and Statistics, 81(4) pp 1-10.

[24] Knox, Stock and Watson (2000) "Empirical Bayes with many predictors

[25] Koop, G. and S. M. Potter (1999), "Bayes Factors and Nonlinearity: Evidence from Economic Time series," J ournal of Econometrics, 88, 251-281.

[26] Koop, G. and S.M. Potter (2001), "The Vector Floor and Ceiling Model,"

[27] Leamer, E., (1978) Specification Searches, Wiley, New York

[28] Leamer, E., (2001) "The Life Cycle of US Economic Expansions," NBER WP\#8192. 
[29] McConnell, M. and G. Perez-Quiros (2000), "Output Fluctuations in the United States:What Has Changed Since the Early 1980s?" American Economic Review.

[30] Pesaran and Potter (1997), "A Floor and Ceiling Model of US Output," J ournal of Economic Dynamics and Control

[31] Potter, S.M. (1995), "A nonlinear approach to US GNP ," J ournal of A pplied E conometrics,

[32] Romer, C. (1994), "Remeasuring Business Cycles," The J ournal of E conomic History, 54(3), 578-609.

[33] Stock, J. and M. Watson (1989), "New Indices of Coincident and Leading Indicators," In O. Blanchard and S. Fischer edited NBER Macroeconomic Annual Cambridge, MIT Press.

[34] Stock, J.H. and M.W. Watson (1999), "Business Cycle Fluctuations in U.S. Macroeconomic Time Series," ch. 1 in J.B. Taylor and M. Woodford (eds.), Handbook of Macroeconomics, Vol. 1, 3-64.

[35] Stock, J.H. and M.W. Watson (2002), "Diffusion Indices J ournal of B usiness and E conomic Statistics, Kennedy School of Government, Harvard University.

[36] Watson, M. (2002), "'Proceeding of Ecconometric Society World Congress 


\section{Spider Chart of Spread Around Troughs}

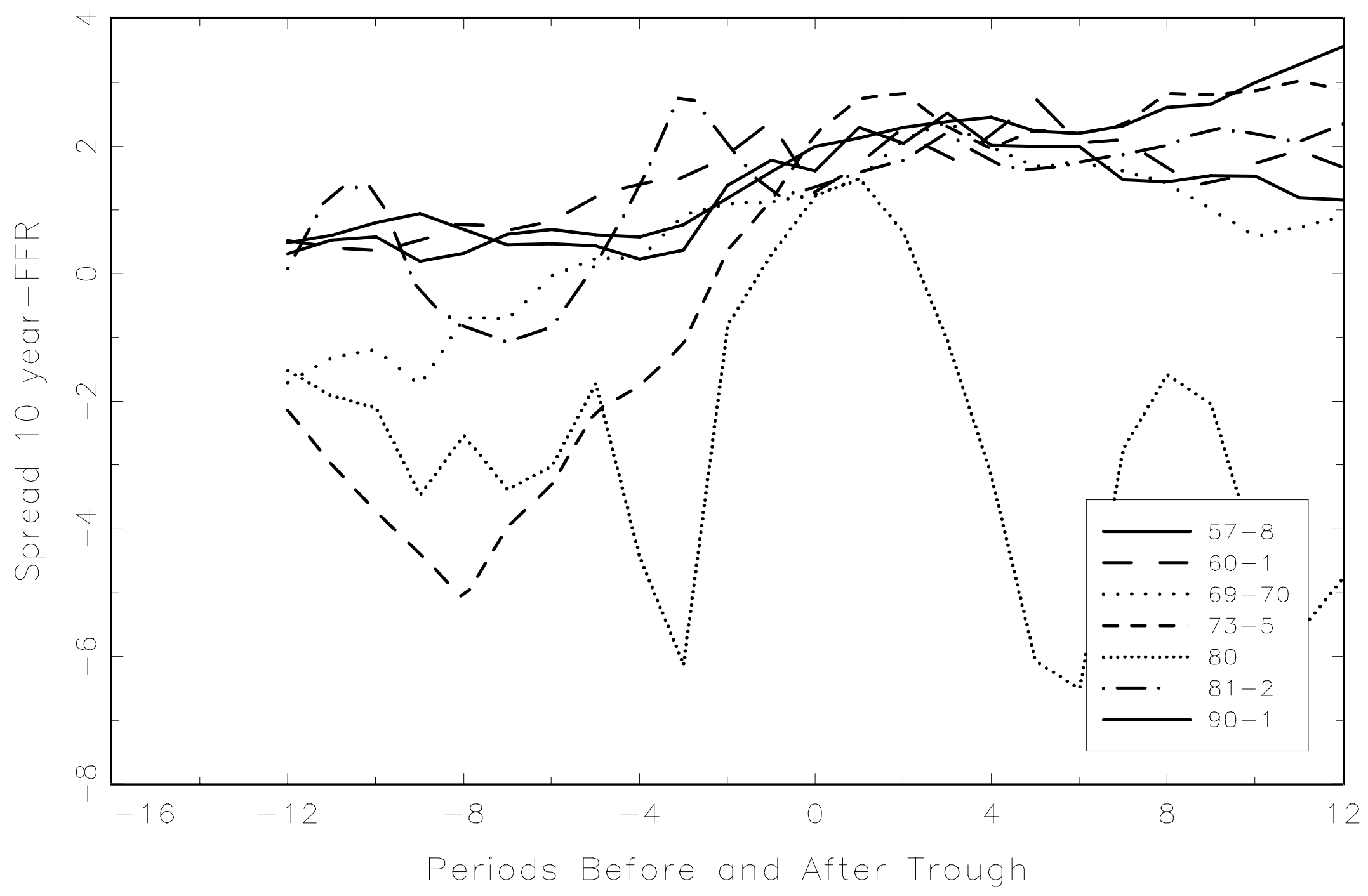


Spider Chart of Unemployment Rate Around Peaks

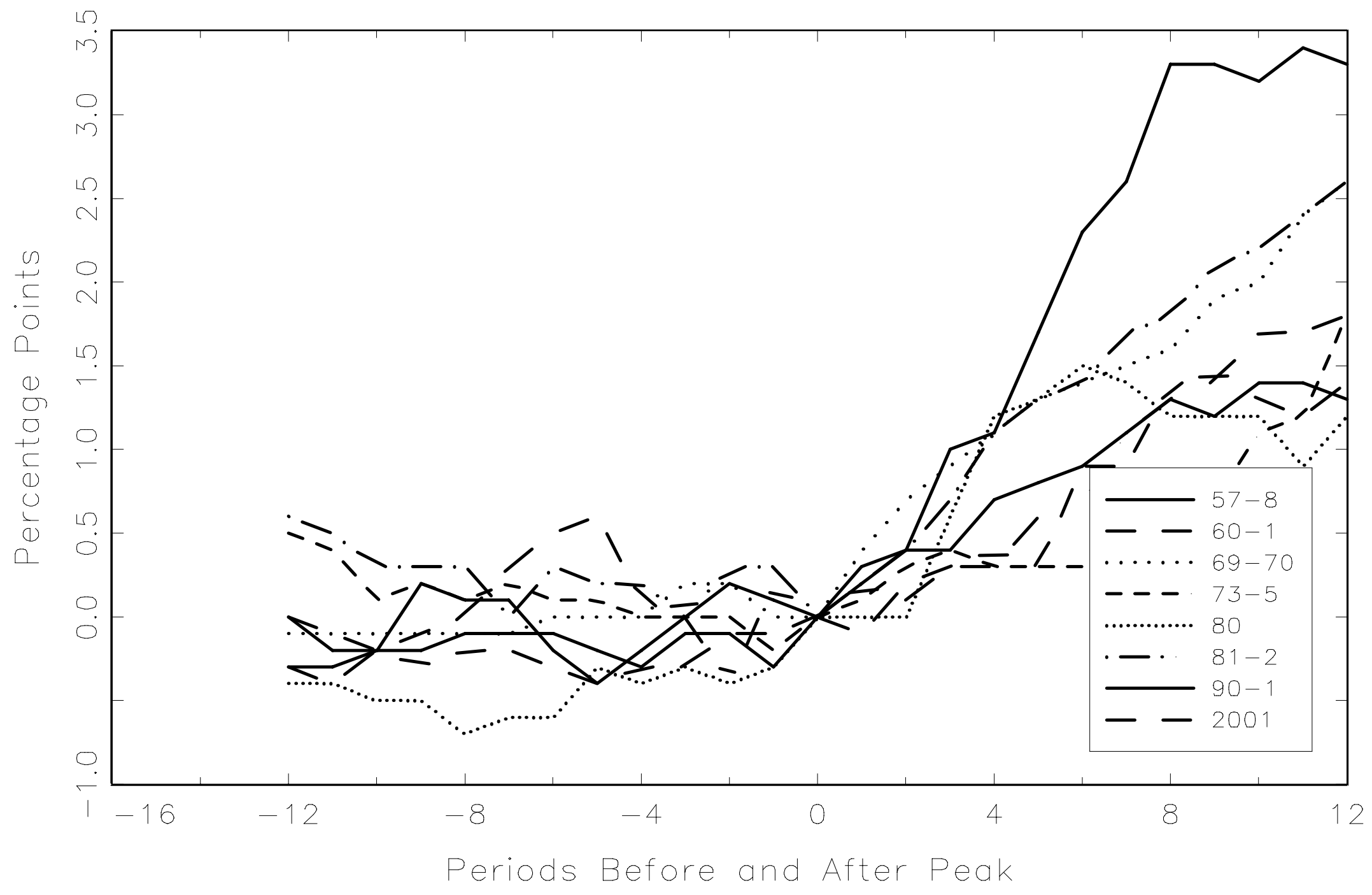


Spider Chart of S\&P Around Peaks

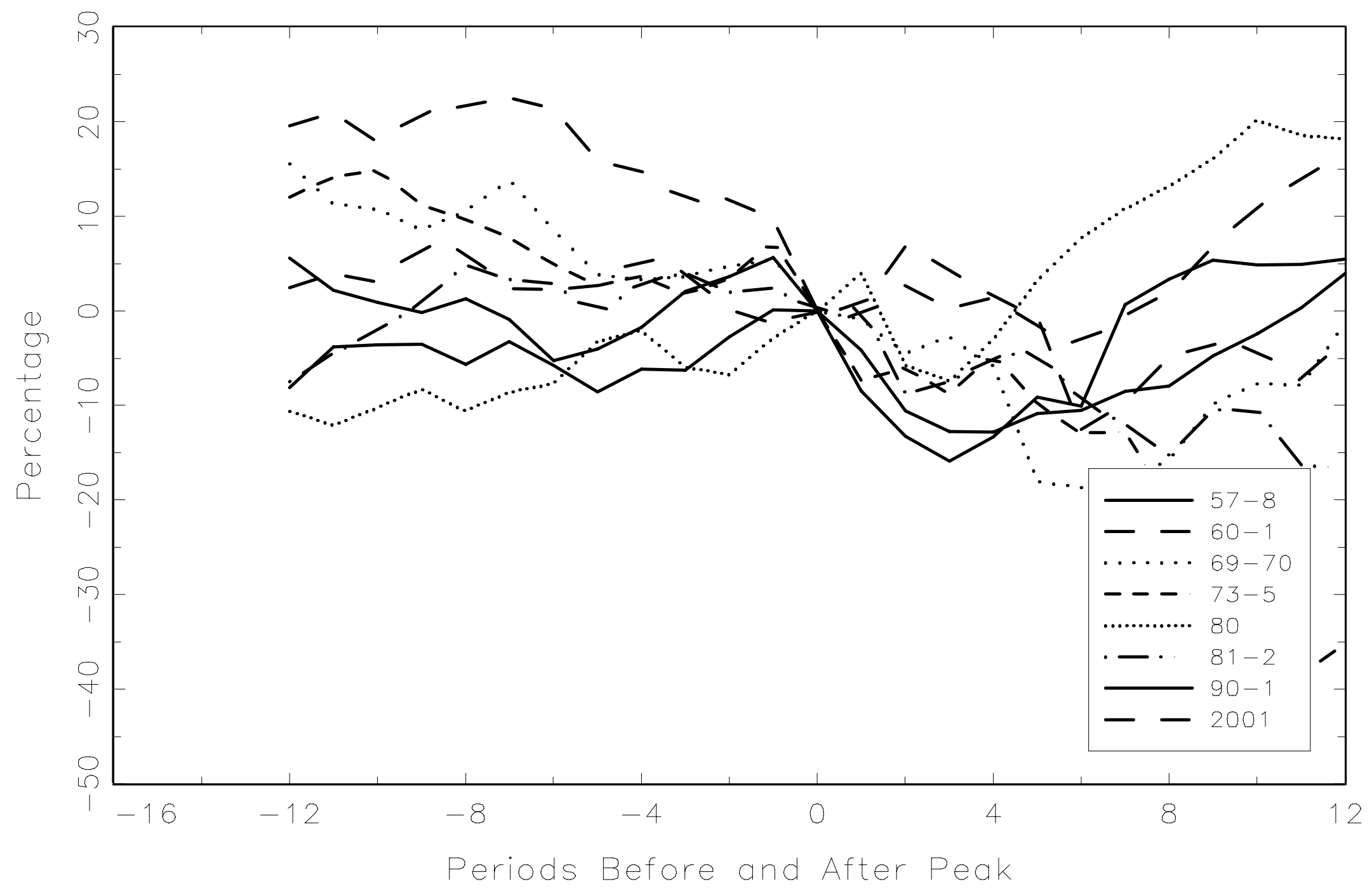




\section{Spider Chart of Unemployment Rate Around Troughs}

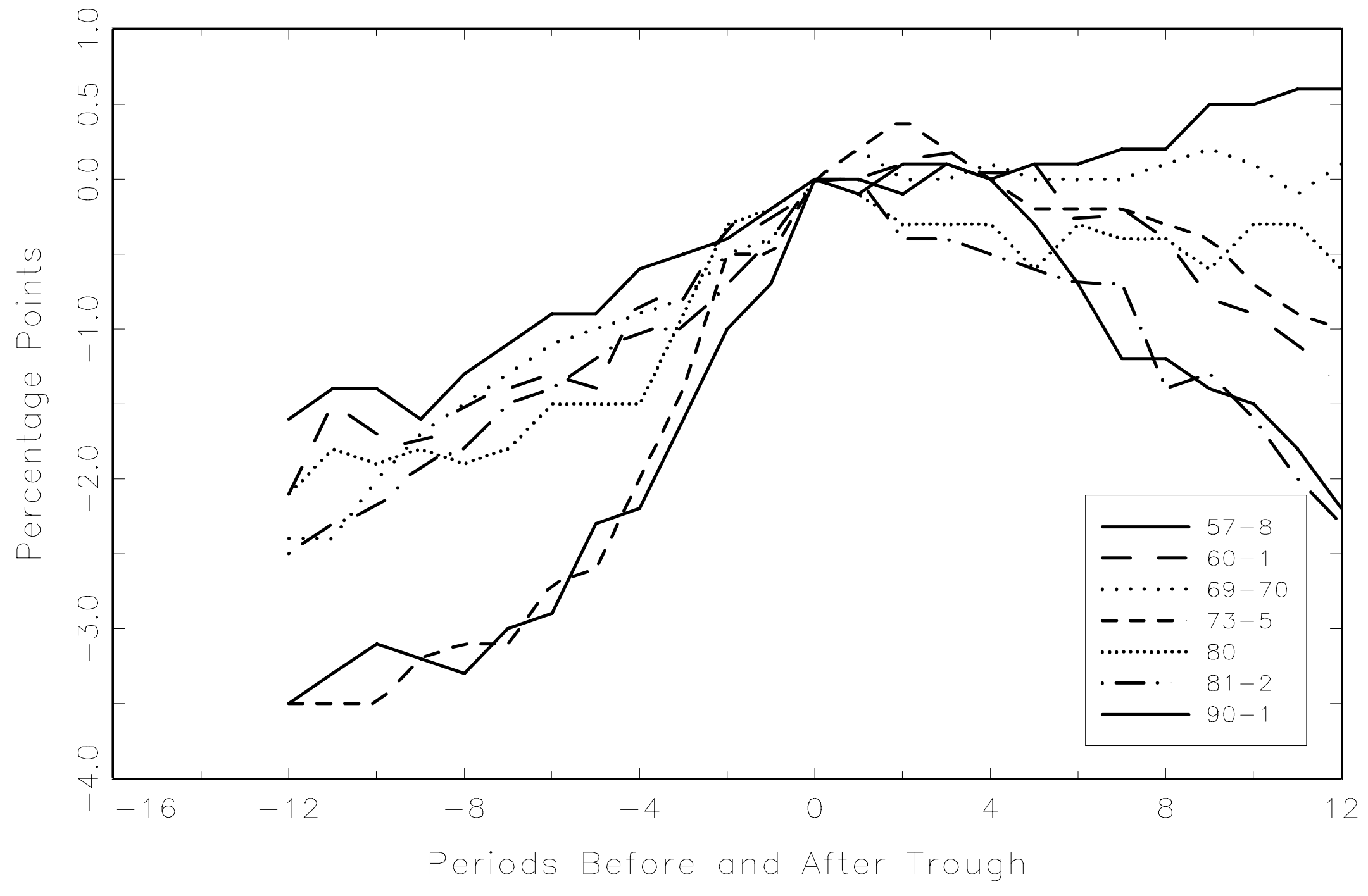


Spider Chart of S\&P around Troughs Assuming December 2001 Trough

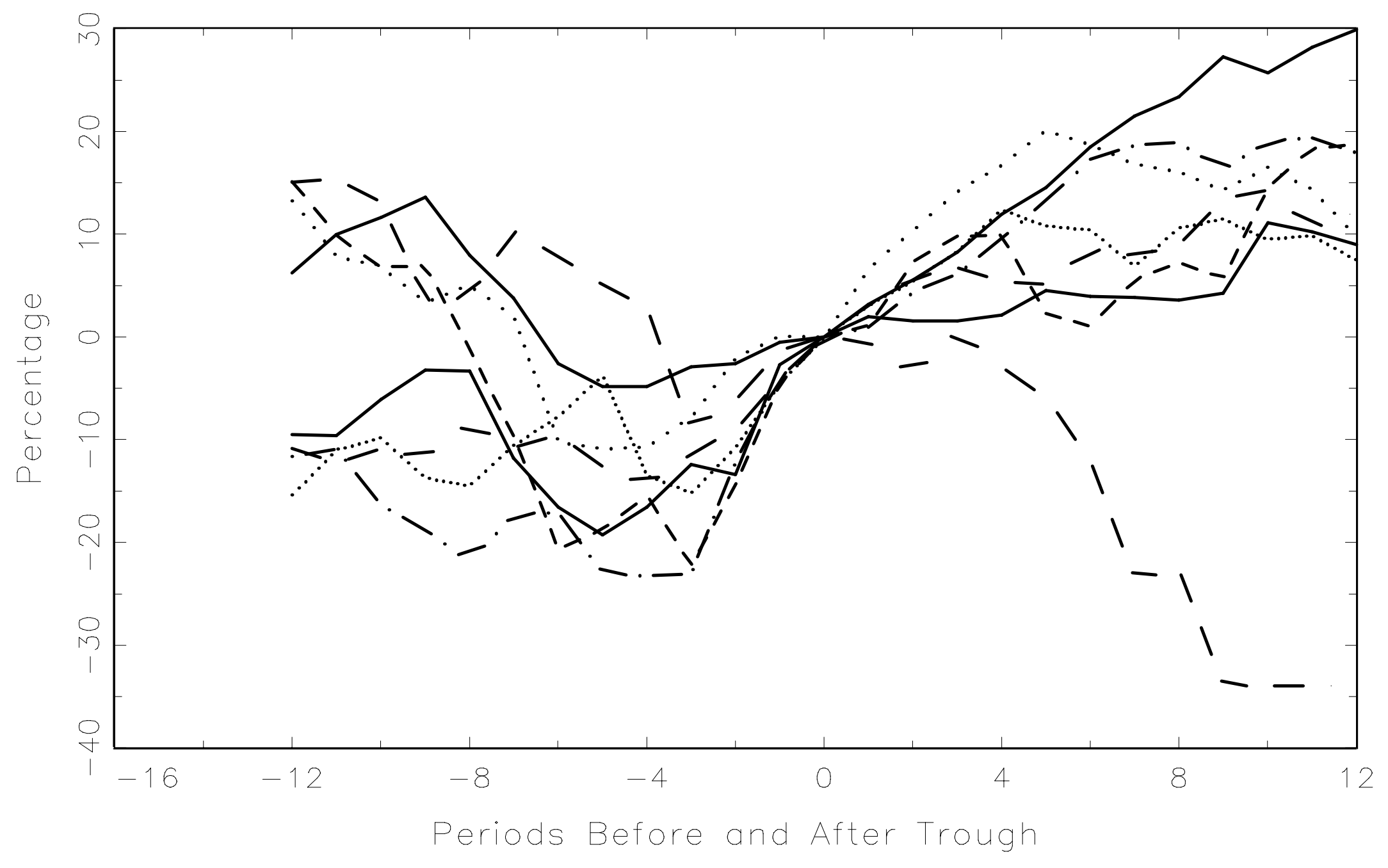


Figure 1: Posterior Mean of Phases

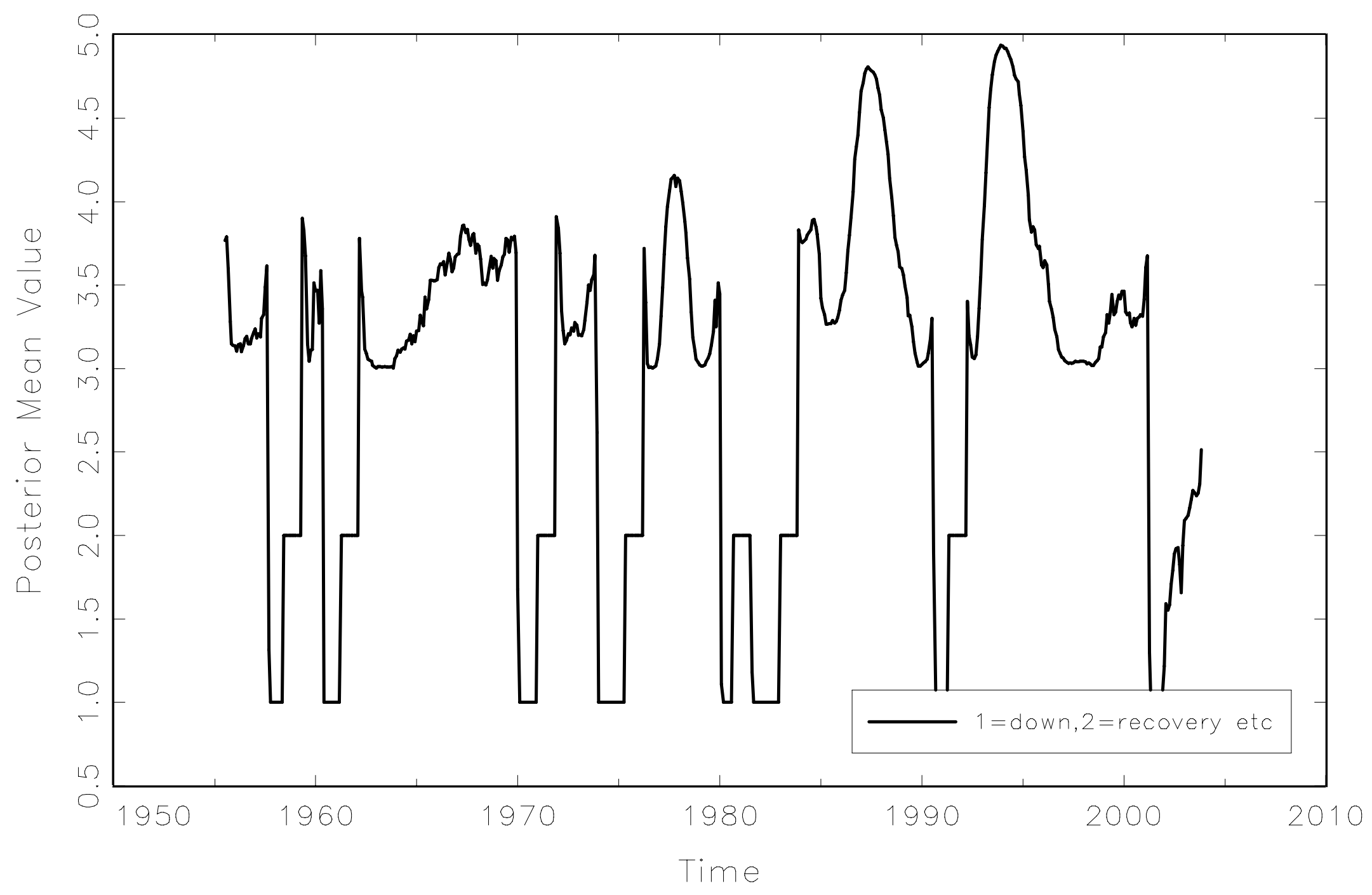


Figure 2: Posterior Probability of Phases

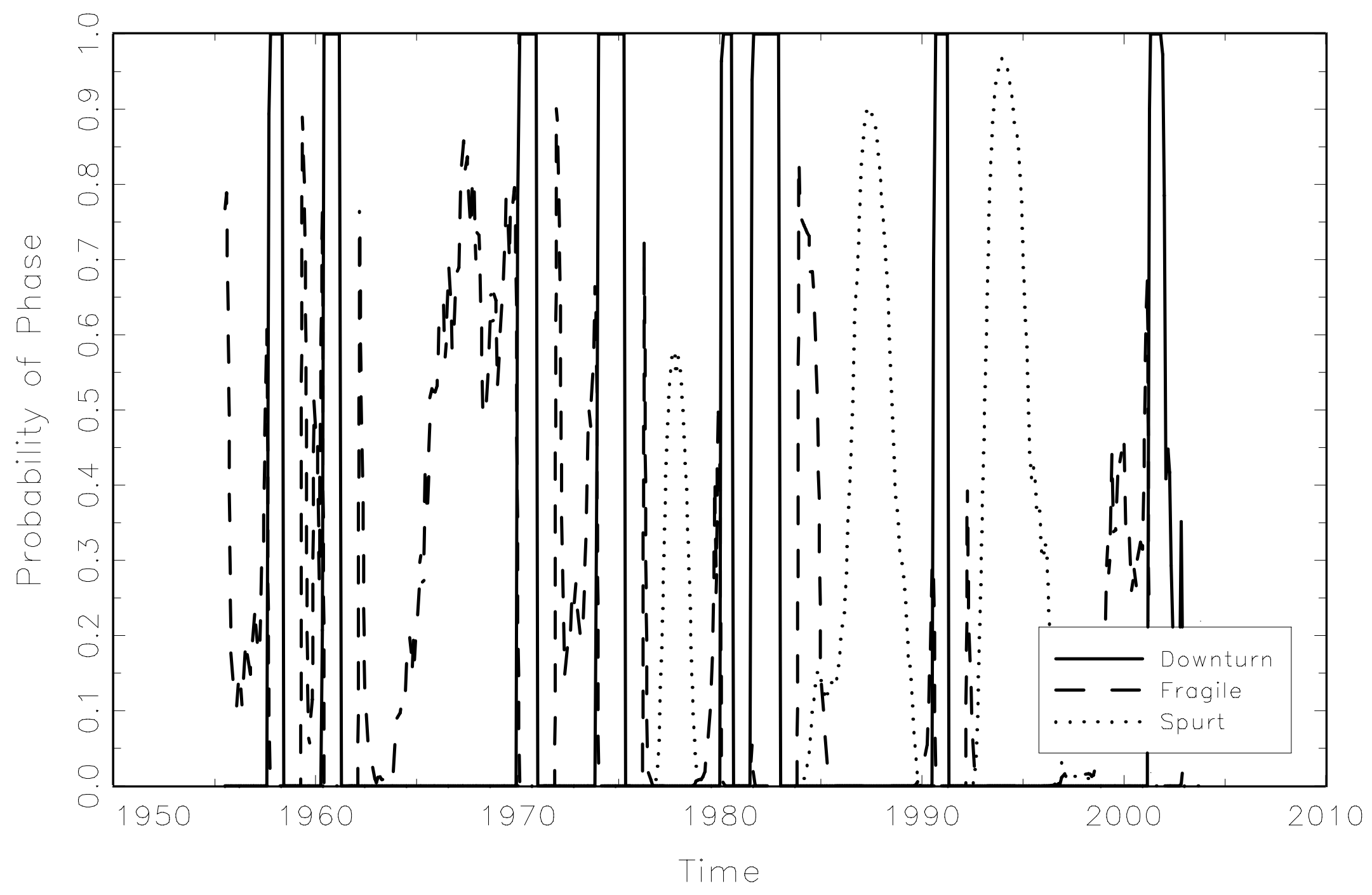


Figure 3: Spread Coefficients

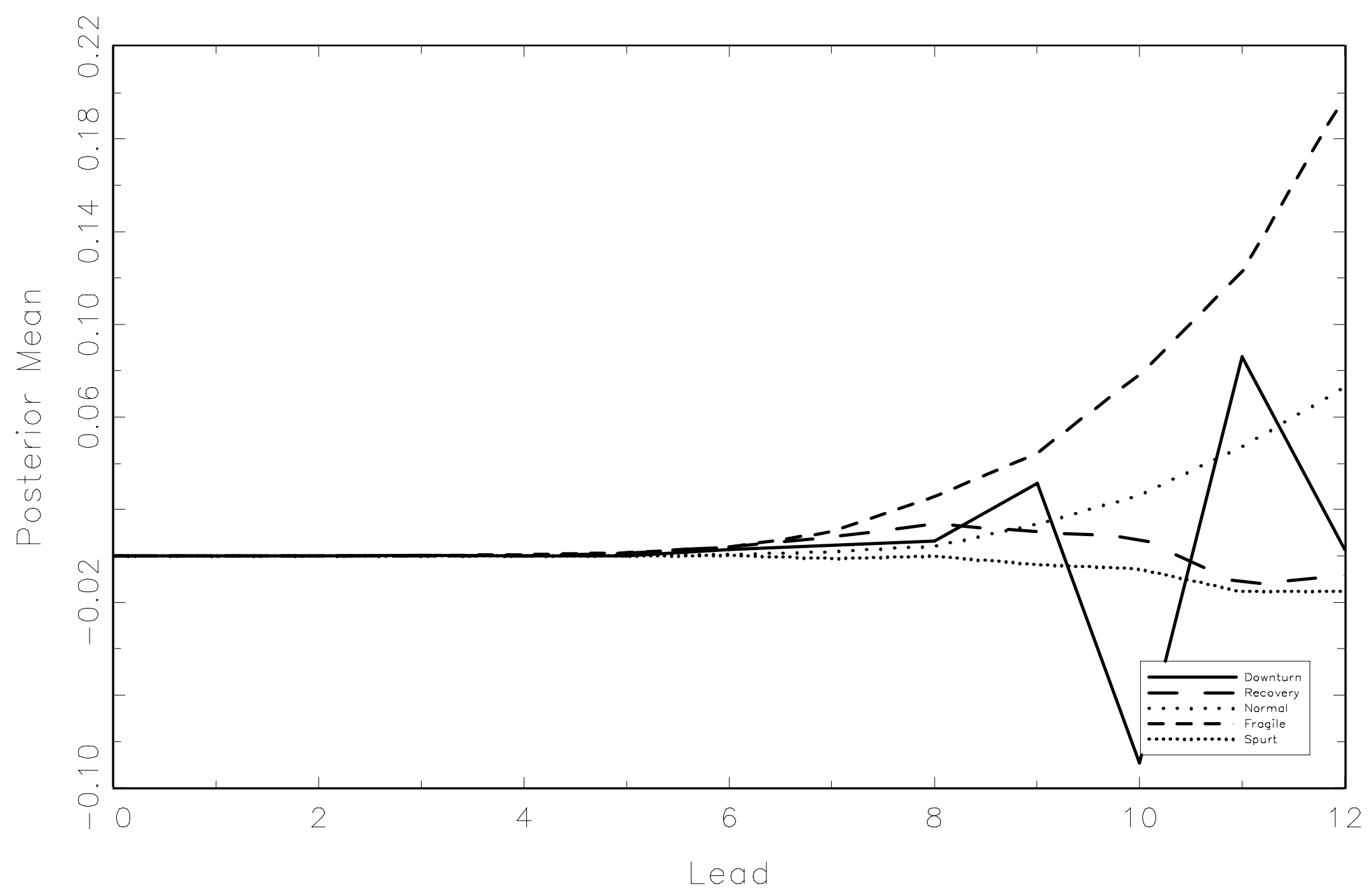


Figure 4: Unemployment Coefficients

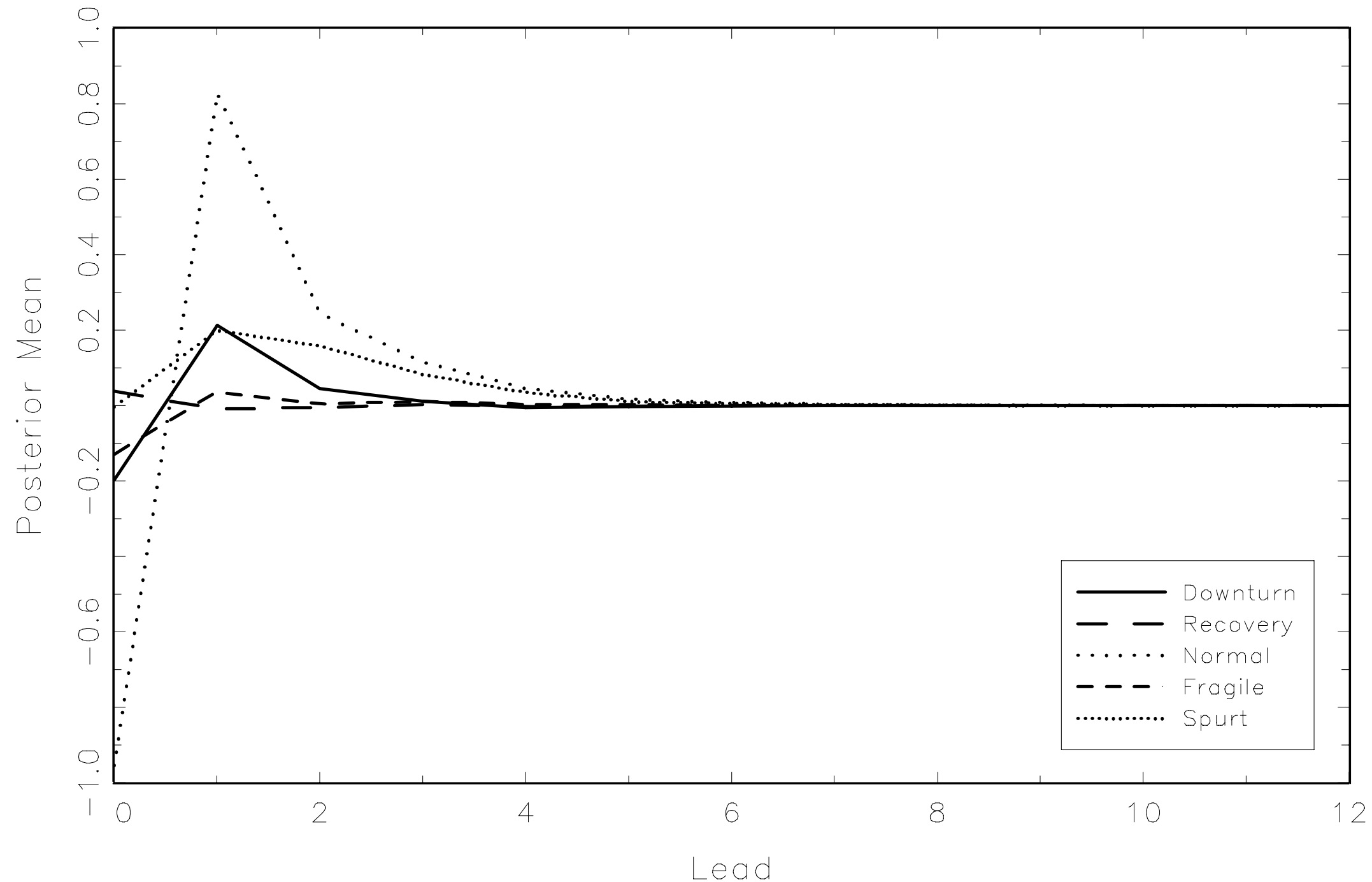


GAUSS Mon Nov 04 13:38:16 2002

Figure 5: Posterior Probability New Business Cycle Started

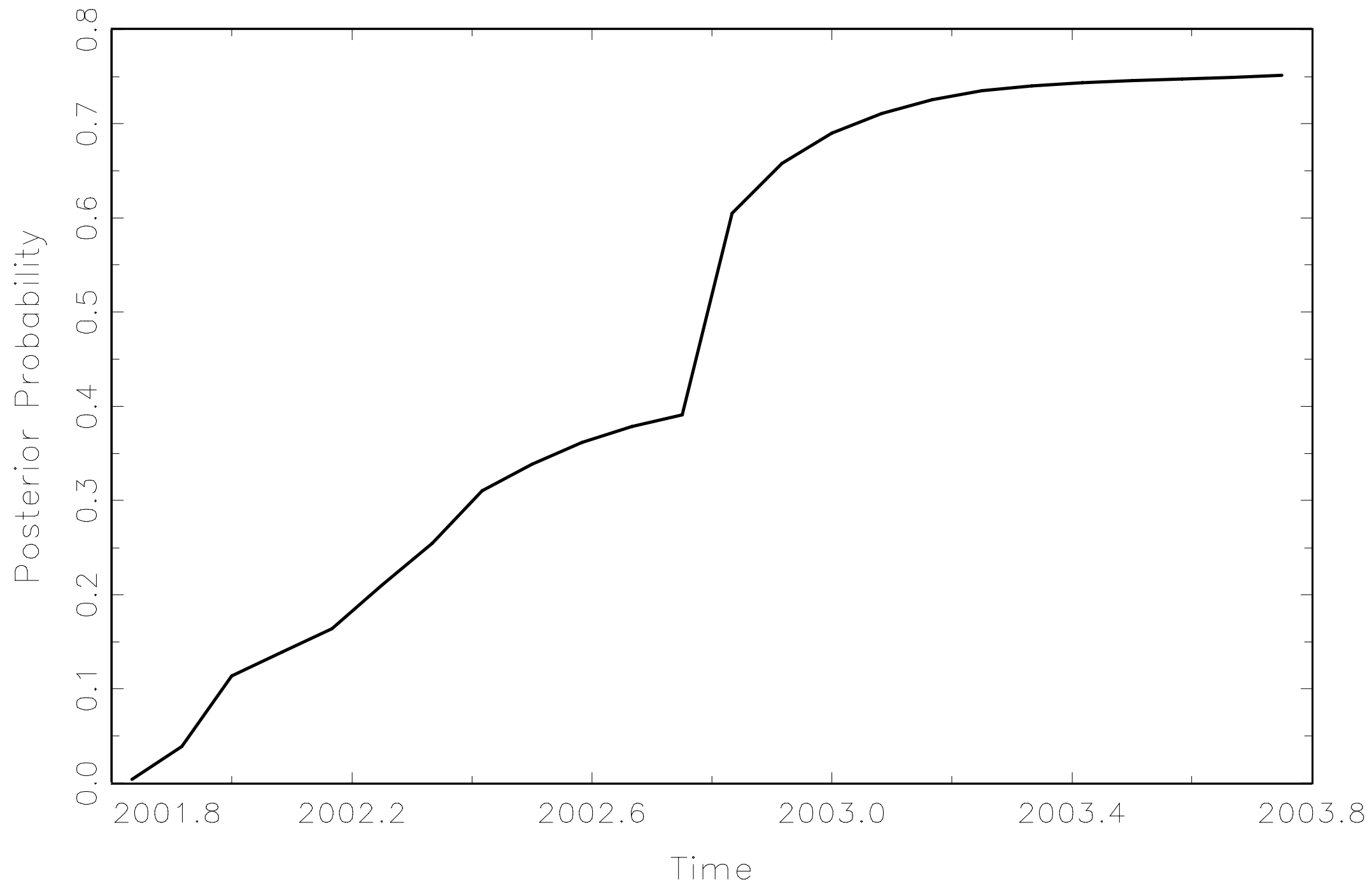


GAUSS Mon Nov 04 13:38:16 2002

Figure 6: Predictive Distribution for Oct 2003 Spread

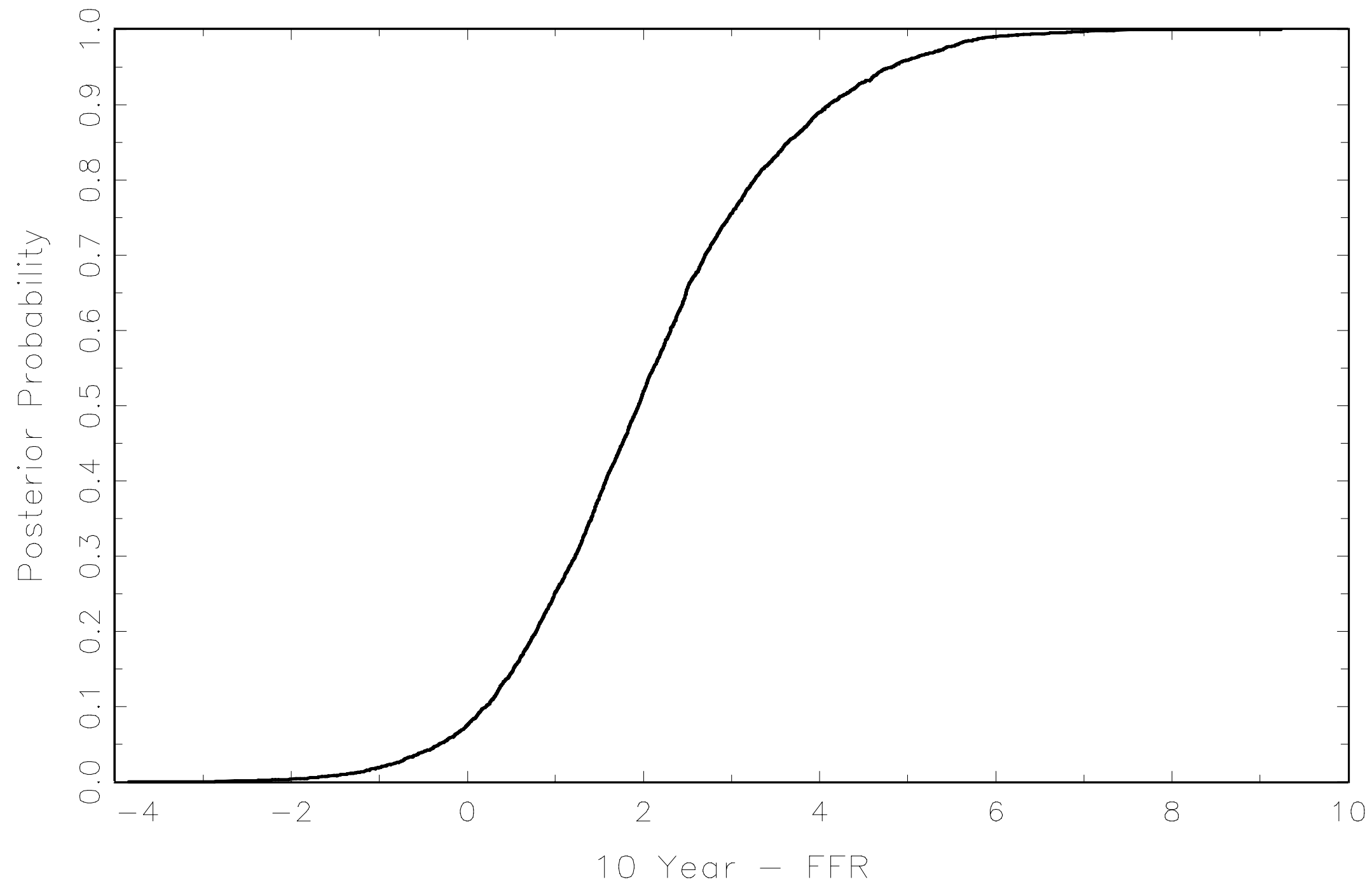


GAUSS Mon Nov 04 13:38:16 2002

Figure 7: Predictive Distribution for Oct 2003 Unemployment Rate

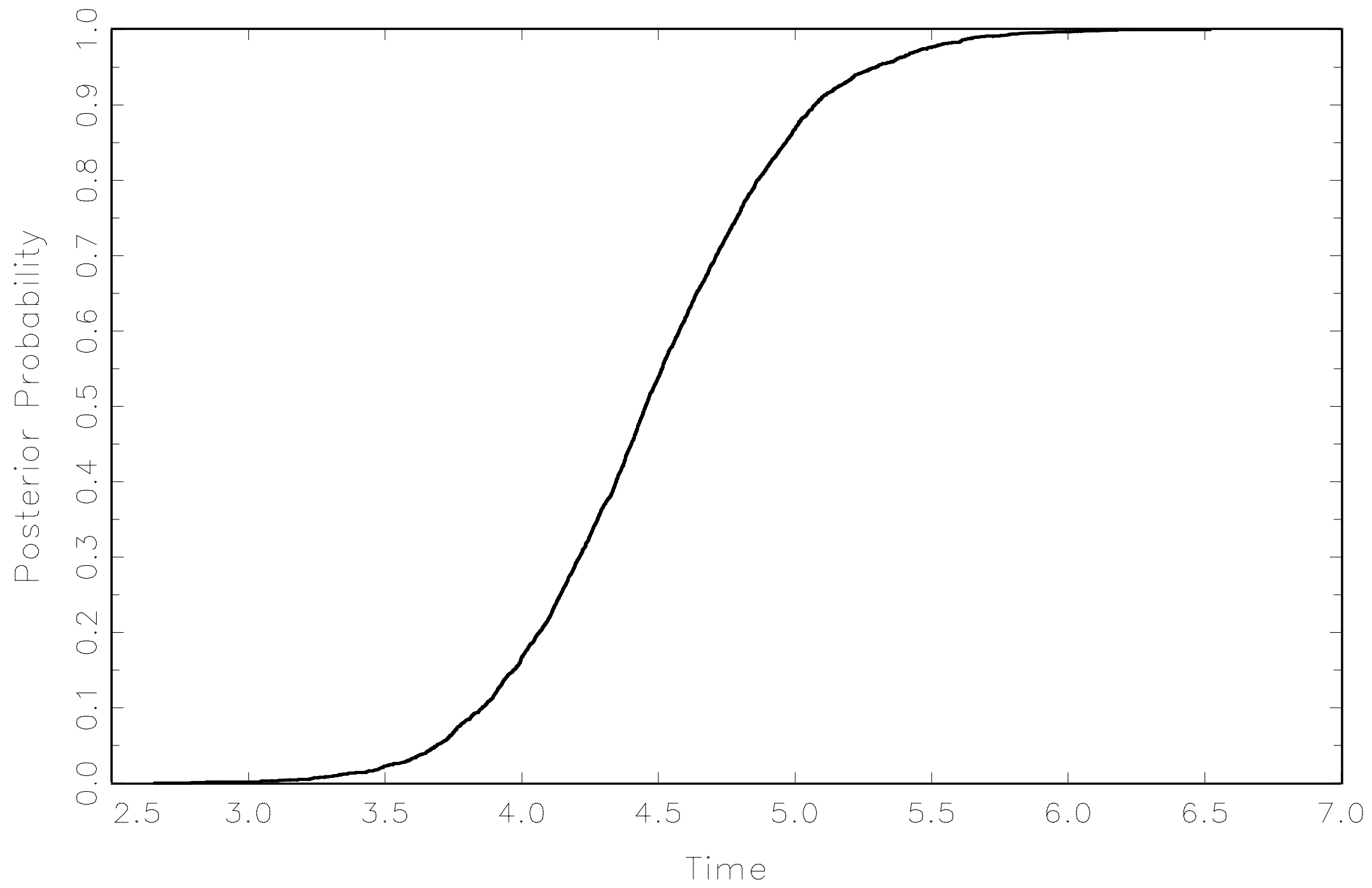

